\title{
Effective elasticity and persistence of strain in active filament-motor assemblies
}

\author{
Arvind Gopinath ${ }^{1,2, \ddagger,}$, Raghunath Chelakkot ${ }^{3, \ddagger}$, and L Mahadevan ${ }^{4, *}$ \\ ${ }^{1}$ Department of Bioengineering, University of California Merced, Merced, CA, USA. \\ ${ }^{2}$ Health Sciences Research Institute, University of California Merced, Merced, CA, USA. \\ ${ }^{3}$ Department of Physics, Indian Institute of Technology Bombay, Mumbai, India. \\ ${ }^{4}$ Department of Physics, Department of Organismic and Evolutionary Biology, and School of Engineering and Applied Sciences, \\ Harvard University, Cambridge, MA, USA. \\ *Correspondence: agopinath@ucmerced.edu, Imahadev@harvard.edu. †Equal contribution
}

\begin{abstract}
Cross-linked, elastic, filamentous networks that are deformed by active molecular motors feature in several natural and synthetic settings. The effective active elasticity of these composite systems determines the length scale over which active deformations persist in fluctuating environments. This fundamental quantity has been studied in passive systems; however mechanisms determining and modulating this length-scale in active systems has not been clarified. Here, focusing on active arrayed filament-motor assemblies, we propose and analyze a minimal model in order to estimate the length scale over which imposed or emergent elastic deformations or stresses persist. We combine a mean-field continuum theory valid for weakly elastic assemblies with high dimensional Multi-Particle Collision (MPC) based Brownian simulations valid for moderate to strongly elastic and noisy systems. Integrating analytical and numerical results, we show that localized strains - steady or oscillatory - persist over well-defined length scales that dependent on motor activity, effective shear elasticity and filament extensibility. Extensibility is key even in very stiff filaments, and cannot be ignored when global deformations are considered. We clarify mechanisms by which motor derived active elasticity and passive shear elasticity of the filamentous backbone combine to effectively soften filaments. Surprisingly, the predictions of the mean-field theory agree qualitatively with results from stochastic discrete filament-motor model, even for moderately strong noise. We also find that athermal motor noise impacts the overall duty ratio of the motors and thereby the persistence length in these driven assemblies. Our study demonstrates how correlated activity in natural ordered active matter possesses a finite range of influence with clear testable experimental implications.
\end{abstract}

\section{INTRODUCTION}

The active strain generated by molecular motors interacting with elastic bio-filaments is the principal mechanism of force generation and motion in cell biology (1-11). In these biological assemblies, elastic bio-filaments are dynamically deformed and strained by localized, steady or time-dependent strains imposed by motors. The strength and intensity of these deformations depend on bio-filament elasticity, the type and density of cross-links, geometric constraints such as due to filament intersections or boundary conditions, and finally motor mechanochemistry. Stochasticity and noise - originating either as thermal fluctuations or via athermal variations in motor activity due to small motor density - further impacts emergent deformation fields. Since temporally varying filament deformations can in turn regulate motor activity as in eukaryotic cilia (12-15), the filament mechanics is ultimately controlled by the two-way nonlinear coupling between motor mechanochemistry and filament elasticity. The length scale characterizing the extent of correlated activity - that is the maximum distance between spatially separated components of the assembles that can still communicate mechanically ultimately enables and limits function and physiology. Previous studies have identified the length scale over which mechanical strains in passive networks decay and have clarified the roles of shear and extensional modes of deformation in controlling this length. Here we analyze the emergence of an analogous decay length in active filament-motor networks.

This suggests that in active arrayed filament-motor assemblies such as in axonemes, one must consider the finite extensibility of microtubules to evaluate the overall bending, shear and extensional elastic effects. These overall bulk properties control the effective softness of the composite material and length scales over which mechanical information may be communicated. A finite decay length implies that any transmission of mechanical information between spatially separated motor aggregates has a limited range, which restricts the lengths over which motor can coordinate their activity. In Fig. 1(a), we show two distinct motor 
(a)

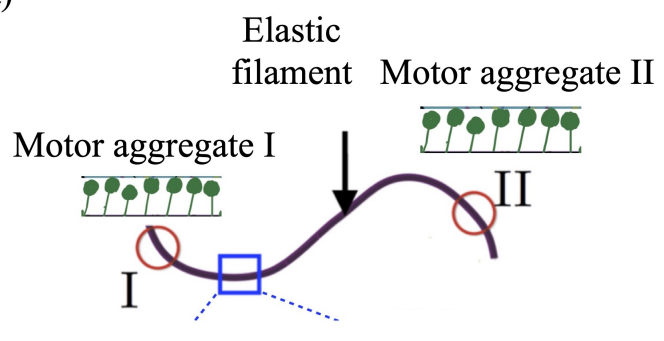

(b)

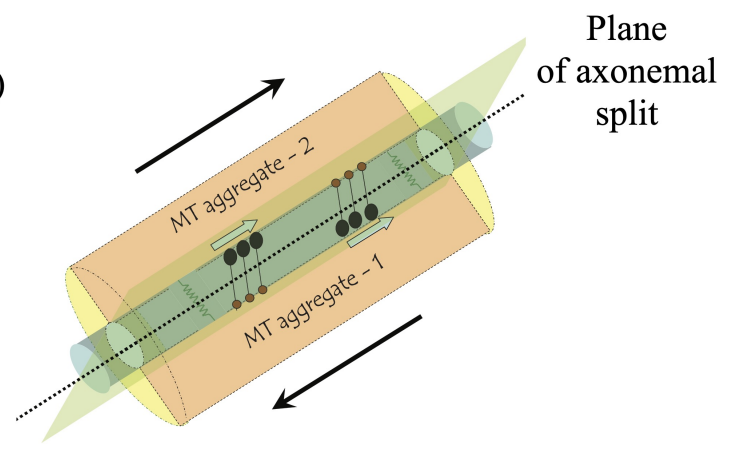

Figure 1: a) An active, motor-filament composite with spatially separated motor aggregates, (I) and (II). The softer the intervening filament segment connecting (I) and (II), the higher the tendency of the motor groups to remain uncoupled and hence not move collectively. (b) Weak mechanical coupling may affect the emergence and control of synchronized motion and oscillations in flagellar axomenes. We show a schematic of the motor generated relative sliding of the two halves of the axoneme that drives oscillations (13-15). If motor aggregates (MT-1 and MT-2) are unable to synchronize, coherent sliding is prevented and oscillations may be localized to parts of the axoneme.

aggregates (patches) labeled I and II cross-linking a pair of thin filaments that may both bend and shear. Assuming the filament is inextensible, mechanical activity by group (I) and shear (sliding) induced by this patch is transmitted by attached motors in (II) over arbitrarily large inter-aggregate distances. However, when the filament is extensible, ability of motors in group (I) to mechanically link with group (II) is limited. For very soft filaments, localized strains and stresses induced at location I lead to propagating fields that decay along the filament, are not sensed by motors at II and thus prevents sets I and II from coordinating. In short, softness leads to de-coherence and stiffness ensures coherence. Additionally, kinetics of motor activity set by ATP hydrolysis rates can couple back to effective extensibility by introducing shear stiffening thus enhancing or disrupting coherence between the separated motor groups. As a concrete illustration of the implications resulting from the finite persistence lengths over which spatially separated motors may communicate, we turn to the example of eukaryotic flagellum (Figure 1(b)). This flexible, slender complex organelle is comprised of ordered assemblies of stiff microtubules, dynein ATPase motors and passive nexin proteins. It has been hypothesized that action of arrayed dynein motors on the microtubules coupled with geometric and elastic constraints convert sliding forces and motions into oscillations with well-defined frequencies and wavelengths $(2,13,16-20,33,34)$. Models with varying degrees of complexity have been introduced to study these flagellar and similar filament-motor assemblies $(3,5-10,20,33,34)$. In these theories, the microtubules are assumed to be inextensible - that is, the extensional deformation along the filament is assumed negligible. This implies that localized extensional strains on the filament are felt all along its contour irrespective of the total length with beating wavelengths predicted to scale as the flagellar length without limit. This deduction is at odds with experimental evidence that indicate that the beating wavelength of flagella are self-limiting even as the flagella themselves range from tens of microns to nearly a centimeter (2-4) . Experiments strongly suggest a substantial degradation of information in eukaryotic flagella, when transmitted over large lengths via mechanical means.

We note that many bio-filaments such as microtubules are intrinsically stiff in the absence of cross-linking filaments. Therefore, filament extensibility has been neglected in most theoretical studies on actively driven filaments. However, several models of passive filament bundles $(7,35-37)$ do account for finite stretching stiffness - the extensional elasticity - of the filaments. These studies suggest that when bundled filaments are bent, the characteristic length scale over which the mechanical information is transmitted is determined by a combination of the effective shear stiffness of the bundle, stretching, as well as the bending stiffness of the individual filaments. In particular, a recent study by Ward et. al (37) on mutually sliding actin filaments suggests that the nontrivial dependence of sliding friction on the filaments' overlap length can be explained by a length-scale arising from the finite extensional rigidity of f-actin.

Motivated by these considerations, we address three principal questions in this paper in reference to active filament-motor assemblies:

1. What is the effective coarse-grained elasticity of composite arrayed active filament-motor assembles?

2. What sets the length scale over which imposed local elastic deformations or imposed localized stress persist in such 
systems? This we study in systems with imposed as well as naturally emergent strains.

3. How do overall elasticity and motor kinetics (such as attachment rates, detachment rates, duty ratio) vary with properties of the constituents and impact persistence length and emergent coordination?

Recognizing that motor groups interacting with an assumed infinitely-stiff filament will immediately coordinate their dynamics, we hypothesize that different components of elasticity - extensional, active or shear softening - can mediate interactions between spatially separated regions of the filament (Figure 1(a)). To test and explore this hypothesis, we analyze analytical and computational models focusing on extensional systems to extract the length scale(s) over which actively generated strains persist. The outline of this paper and a summary of the main results are as follows:

1. In section 2, we elucidate the coupling between bend, shear and extensional elastic components in a passive composite containing filaments and passive crosslinkers. This provides a theoretical framework building on which active assemblies can be analyzed. We obtain the persistence of extensional and bending strains for a passive composite.

2. In section 3 we use these results as the foundation to understand the noise-free, mean-field model for active filament-motor composite. Focusing on the role of shear stiffening and strain softening in extensional systems we derive persistence lengths of extensional strain for a) filaments in rigor, b) an active filament that is stationary with strain coupled to motor activity, and c) the persistence length when the filament is subject to localized static or oscillatory strain. The mean-field theory provides analytical expressions for this length-scale in the frequency-locked homeostatic limit; we find that length-scale is dependent on motor mechanochemistry, motor elasticity, and filament extensibility. The persistence lengths derived in b) and c) provide insights into when spatially separated and independently oscillating motor domains may synchronize elastically.

3. The theoretical analysis provides a baseline understanding of the underlying physics, albeit with limitations. First, it does not shed light on what happens when the number of motors is small or when discrete noise due to motor kinetics is significant. Indeed, the onset of steady or oscillatory extension necessarily involves small numbers of motors when the deformation field develops from this initially noisy state. In section 4, we analyze a discrete stochastic numerical model that incorporates athermal motor noise arising due to discrete nature of motor kinetics and the finite number (density) of motors. Surprisingly, the predictions of the mean-field theory agree qualitatively with the results from stochastic discrete filament-motor model, even for moderately strong noise. For strong athermal motor noise, we predict rich dynamical features, including the modification of the motor duty-ratio, and localized regions of coherent oscillations.

\section{MEAN-FIELD MODEL FOR PASSIVE ASSEMBLIES}

We start our analysis by studying a passive filament assembly (composite) comprised of two filaments that are connected by permanent elastic cross-linkers. This composite therefore has bending, shearing and extensional moduli, which are all mutually coupled via the geometry of the composite.

In our model, two elastic strips of unstrained length $\ell$, lateral width $b$ and thickness $w \ll \ell$ are held at a distance $D \ll \ell$ apart by a series of passive, linearly elastic springs with areal density $\rho_{\mathrm{N}}$ and stiffness $k_{\mathrm{N}}$ (Figure 2(a) specialized to the no-motor case). The passive springs are compliant in shear along the axial direction as shown. They are however laterally stiff, preventing any change in the gap thickness $D$. Motivated by the high aspect ratio, slender filaments abundant in biology, we restrict ourselves to the limiting case $\ell \gg b \gg \max (w, D)$. Finally to focus on the essential physics, we allow only for planar two-dimensional extensional, sliding and bending deformations of the composite filament. Thus deformation and stress fields may be reduced to one dimensional fields along the axial direction.

At length scales large compared to the spacing between the passive springs, the composite acts as a filament of thickness $D$, with an effective shear modulus that depends on $D$ and on the areal density $\rho_{N}$ and elastic constant $k_{N}$. Suppose a shear stress $\sigma_{s}$ is acted upon the top filament with surface area $b \ell$, causing a sliding displacement $\Delta$ between both the filaments. This applied stress is balanced by the restoring elastic stress exerted by the connecting springs $\rho_{\mathrm{N}} b \ell$ leading to the force balance relation, $\sigma_{s} b \ell \sim \Delta k_{\mathrm{N}} \rho_{\mathrm{N}} b \ell \sim G \Delta b \ell$. Th effective shear modulus of the whole strip that includes contributions from these passive springs is $G^{*} \sim D k_{\mathrm{N}} \rho_{\mathrm{N}}$. For a strip of lateral width $w$, we have the stiffness $G^{*} w / D \sim w k_{\mathrm{N}} \rho_{\mathrm{N}}-$ consistent with classical result for a passive cross-linked railway track model.

To further analyze the general extensional and bending deformations of this composite filament, we choose a local coordinate system for the filament, characterized by an arc-length variable $s$ so that the filament is in the range $s \in(0, \ell)$. Consider two points $\mathbf{r}_{1}$ (on filament 1) and $\mathbf{r}_{2}$ (on filament-2) as shown in Fig. 2(a) (lowest tile) that face each other when the filaments are in the undeformed state. We define slide $\Delta(s)$ as difference between $\mathbf{r}_{1}$ and $\mathbf{r}_{2}$, relative to its initial difference before deformation. Any arbitrary deformation of the filament can be decomposed into three principal modes - bending, shear and 
(a)

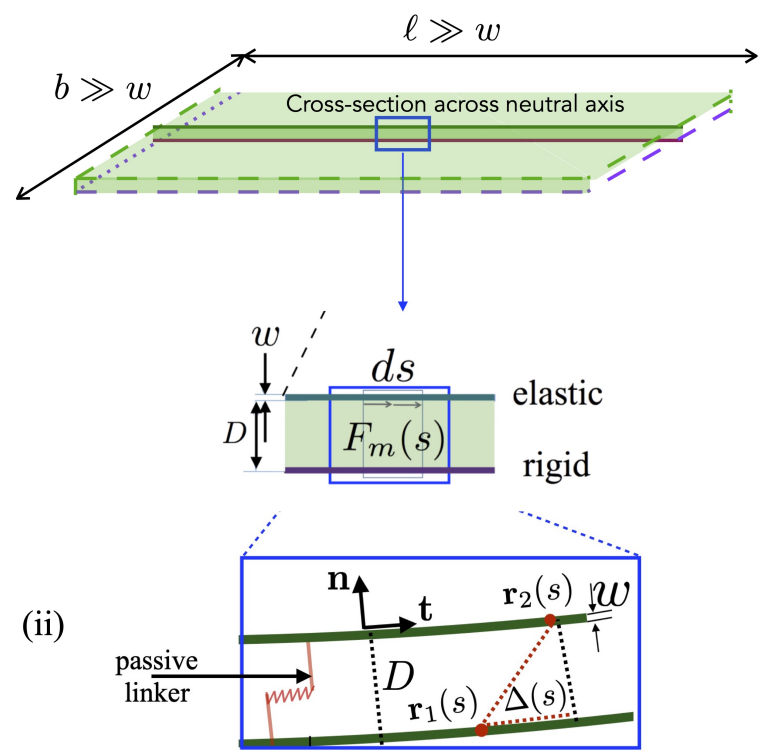

(b)

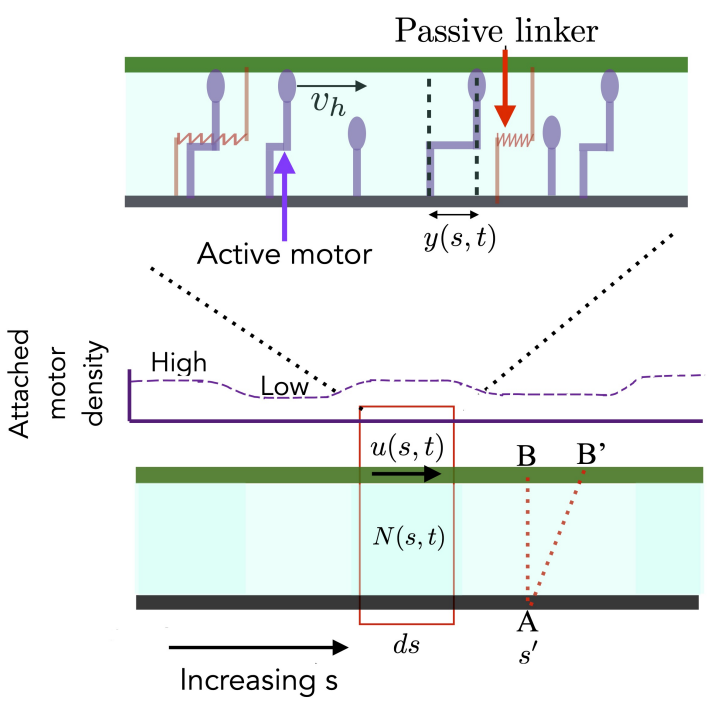

Figure 2: (a) (i) (Top) Cross-section of active motor-filament composite strip. The bottom surface (purple dashed lines) is rigid and fixed. The upper surface (green dashed line) can stretch and slide but cannot bend. We choose $w \ll \ell$ and $w \ll b$. (Bottom) We show a close-up of the blue box from the top tile. The composite filament is comprised of a thin extensible sheet of width $w$ and lateral width $b \gg w$ that is a distance $D$ above an inextensible substrate. This distance is spanned by active motors generating an active force density $F_{m}$. Deformation fields vary spatially in the axial direction shown. The direction normal to the plan of the paper is the neutral direction. (ii) Schematic of the composite passive strip analyzed in the mean free model. The thickness of the strip $w$ is much smaller than the length $\ell$ and width $b$. Variables used in the theory are shown. (b) (i) Further magnifying the composite filament illuminates the discrete elements comprising the continuous filament. Shown are the passive links (red) and active motors (purple) spanning the distance $D$. Motors attach with a pre-extension $d_{m}$ and then move along the upper filament with speed $v_{h}$ relative to their base. The mechanochemical state of attached motors is quantified by their internal extension $y$. Coarse-graining motor activity provides the local density of attached motors $N(s, t)$ and the local mean motor extension $Y(s, t)$ which my be used to estimate the local active force densities $F_{m}(s, t)$. and the local filament displacement is $u$. (ii) Variations in the motor density and the motor extension coupled with passive extensional elasticity of the filament (green) and shear elasticity of the linkers (red) influence how material point $B$ on the filament moves to $B^{\prime}$ in response to forces.

stretching characterized by three scalar fields . These fields are however not all independent. The angle made by the tangent to the centerline, $\theta(s)$ is then related to the slide $\Delta(s)$ and the displacement $u(s)$ by geometrical constraints. The local geometry along the filament pair is characterized by the relations $d \mathbf{r}_{1} / d s=\mathbf{t}, d \mathbf{t} / d s=C \mathbf{n}$ and $d \mathbf{n} / d s=-C \mathbf{t}$, where $\mathbf{n}$ and $\mathbf{t}$ are the normal and tangent vectors at $\mathbf{r}_{1}$ (or equivalently at material point located at $s$ ), and $C=d \mathbf{t} / d s \cdot \mathbf{n} \approx d \theta / d s$ is the local curvature. As the filament deforms, the location of $\mathbf{r}_{2}$ relative to $\mathbf{r}_{1}$ changes due to the deformation. To further the analysis, and without loss of generality, we specify that the composite deforms such that the end at $s=0$ is locally flat - i.e, we fix the tangent angle at $s=0$ to be zero. Then using $(D+w) / \ell \ll 1$, we obtain to leading order

$$
\begin{aligned}
\mathbf{r}_{2}-\mathbf{r}_{1} & \approx D \mathbf{n}+u \mathbf{t}, \\
\frac{d \mathbf{r}_{2}}{d s} & \approx \mathbf{t}-D C \mathbf{t}+\frac{d u}{d s} \mathbf{t}+u C \mathbf{n} .
\end{aligned}
$$

For small deformations, we can assume that all quadratic terms are small so that $u C \approx 0$ in (2). Taking $\left|d \mathbf{r}_{1} / d s\right|=\mathbf{t}=1$, we find

$$
\left|d \mathbf{r}_{2} / d s\right| \approx 1-D \frac{d \theta}{d s}+\frac{d u}{d s} .
$$

Using the relation $d \Delta / d s=\left|\left(d \mathbf{r}_{1} / d s\right)\right|-\left|d \mathbf{r}_{2} / d s\right|$, then yields a simplified version of equation 2 that relates the spatial variation in the shear displacement to spatial variations in bending and stretching

$$
\frac{d \Delta}{d s}=D \frac{d \theta}{d s}-\frac{d u}{d s}
$$


Integrating (3) from $s=0$ to $s$ using the boundary condition $\theta(0)=0$ yields the relationship between shear, extensional and bending deformations,

$$
\Delta(s)=D \theta(s)-u(s)
$$

Since we consider shear, extensional and bending elastic compliances of the filament, we can define an elastic energy functional E

$$
E=\frac{b}{2} \int_{0}^{\ell}\left(B_{\mathrm{pas}}\left(\frac{d \theta}{d s}\right)^{2}+K_{\mathrm{pas}}\left(\frac{d u}{d s}\right)^{2}+G_{\mathrm{pas}} \Delta^{2}\right) d s .
$$

Here, $B_{\mathrm{pas}} \sim E w^{3}, K_{\mathrm{pas}} \sim E w$ and $G_{\mathrm{pas}} \sim \rho_{\mathrm{N}} k_{\mathrm{N}}$ are the passive bending, stretching and shear moduli, and $E$ is the Young's modulus of the material. The Euler-Lagrange equations obtained by minimizing the functional (5) leads to force-balance relations that provide static solutions of a deformed filament, which we now derive in three useful limits.

1. Persistence length for pure extension: When the bending stiffness $B_{\text {pas }} \rightarrow \infty, d \theta / d s \rightarrow 0$ and the filament deforms due to shear and extension alone resulting in the constraint $\Delta \approx-u$. Minimization of (5) yields

$$
\frac{d^{2} \Delta}{d s^{2}}-\left(\frac{G_{\mathrm{pas}}}{K_{\mathrm{pas}}}\right) \Delta=0
$$

which provides the relaxation length scale for pure extension $\ell_{E}^{2} \equiv K_{\mathrm{pas}} / G_{\mathrm{pas}} \sim E w / \rho_{\mathrm{N}} k_{\mathrm{N}}$.

2. Persistence length for pure bending: In the limit of inextensible filaments, $K_{\mathrm{pas}} \rightarrow \infty$ and $d u / d s \rightarrow 0$. Using the relation $\Delta \approx D \theta$ in this limit and minimizing (5) yields the equation

$$
\frac{d^{2} \theta}{d s^{2}}-\left(\frac{D^{2} G_{\mathrm{pas}}}{B_{\mathrm{pas}}}\right) \theta=0,
$$

thus yielding the persistence scale for pure bending $\ell_{B}^{2} \equiv B_{\mathrm{pas}} / D^{2} G_{\mathrm{pas}} \sim E w^{3} / D^{2} k_{\mathrm{N}} \rho_{\mathrm{N}}$. Physically, this implies that as the density of passive links or their stiffness increases, the persistence length associated with purely bending modes decreases.

3. Persistence length for general case: Finally, we consider the general case when both bending and stretching modes are allowed. Allowing for sliding deformations at $s=0$, we repeat the minimization procedure to obtain the two coupled equations,

$$
B_{\mathrm{pas}} \frac{d^{2} \theta}{d s^{2}}+D K_{\mathrm{pas}}\left(D \frac{d^{2} \theta}{d s^{2}}-\frac{d^{2} \Delta}{d s^{2}}\right)=0, \quad \text { and } K_{\mathrm{pas}}\left(\frac{d^{2} \Delta}{d s^{2}}-D \frac{d^{2} \theta}{d s^{2}}\right)-G_{\mathrm{pas}} \Delta=0
$$

corresponding to $\delta E_{T} / \delta \theta=0$ and $\delta E_{T} / \delta \Delta=0$ respectively. Eliminating $\theta$ from the pair of equations in (8) we find,

$$
\frac{d^{2} \Delta}{d s^{2}}-\frac{\Delta}{\ell_{*}^{2}}=0 \quad \text { with } \quad \ell_{*} \equiv \frac{\ell_{B} \ell_{E}}{\sqrt{\ell_{B}^{2}+\ell_{E}^{2}}} \sim\left[\frac{w / D}{\sqrt{1+w^{2} / D^{2}}}\right]\left(\frac{K_{\mathrm{pas}}}{G_{\mathrm{pas}}}\right)^{\frac{1}{2}}
$$

hereby implying that variations in the sliding displacement $\Delta$ are associated with a persistence (decay) length $\ell_{*}$.

The decay length $\ell_{*}$ in equation (9) depends on geometrical properties (square brackets) and elastic properties (parentheses) and has implications for composite filaments that are subject to localized displacements at one end and also to composite assemblies that have internal localized imposed or emergent forces. Filaments that are extremely stiff and resist extension (such as microtubules) may ultimately be forced to deform in the presence of cross-linkers. Increasing either the density of the passive springs (blue) or their stiffness leads to increased shear resistance per unit length. This effectively renormalizes the bending modulus and the extensional modulus. The generality of the framework suggests that similar length scales should appear in a variety of soft systems where bending, shear and extension are coupled, as indeed has been observed more than 75 years ago in both microscopic and macroscopic contexts $(38,39)$.

To put the discussion in perspective, we consider typical parameters for a canonical ordered active composite - the axoneme seen in sperm flagella. We take $w$ and $D$ as the radius and the spacing of microtubules, $E$ as the Young's modulus of microtubules, $k_{\mathrm{N}}$ as the stiffness of passive (nexin) links and $\rho_{\mathrm{N}}$ as the density of these links. Using $w \sim 20 \mathrm{~nm}, D \sim 40 \mathrm{~nm}(12), E \sim 1.2 \mathrm{GPa}$ $(3,12), k_{\mathrm{N}} \sim 16-100 \mathrm{pN} \mu \mathrm{m}^{-1}(40)$ and $w \rho_{\mathrm{N}} \sim 10^{5}-10^{7} \mathrm{~m}^{-1}(40,41)$, we estimate $\ell_{E} \sim 200-500 \mu \mathrm{m}$ while $\ell_{*} \sim 80-200$ $\mu \mathrm{m}$. This estimate is however with just the passive cross linkers contributing to the shear stiffness and therefore values should be treated as an upper limit. Motor activity will naturally influence this scale as attached motors contribute to the instantaneous shear resistance. Assuming that all motors are bound, with linear density $O\left(10^{8}\right) \mathrm{m}^{-1}$ and effective spring stiffness of $10^{-3}$ $\mathrm{N} / \mathrm{m}$, we obtain $\ell_{E} \sim 5-10 \mu \mathrm{m}$. Since sperm flagella are often much longer than this scale (3), the role played by activity in modulating persistence lengths can be crucial and important. 


\section{MEAN-FIELD MODEL FOR ACTIVE ASSEMBLIES}

The composite filament we analyze in the mean-field model comprises of two cross-linked filaments that are held together as in Figure 2(a), except that now the gap spanning the filaments also has active molecular motors with density $\rho_{m}$. These molecular motors are grafted to the lower surface and attach the upper surface periodically as illustrated in Fig. 2(b). We treat the active motors as unidirectional motors with a clear polarity. The filament may be modeled as a track that generates an interaction potential. Attached motor can undergo conformation changes when attaching as seen for cross-bridge systems (9). Additionally, when attached they can also walk relative to the track (10). This composite formalism allows us to model motor-track interaction in a general fashion. Finally, without loss of generality, we assume that the lower filament is rigid (inextensible) while the upper filament remains weakly extensible.

\subsection{Force balance relates extensional deformation of composite assembly to active forces}

In the composite framework for the motor, we envision each motor behaves elastically as an active linear spring $(9,10)$ with spring constant $k_{m}$ and zero rest length. Motors are characterized by an internal variable, the extension $y(s, t)$, that serves as an indicator of how much each motor is stretched. Motors attach in a pre-extended state with an (initial) expected extension $d_{m}$ (expectation value of motors that are attaching/just attached as in (9)). Once attached, in order to relax the pre-stress, the head slides along the filament with a motor-strain dependent speed, resulting in a changing motor extension $y(s, t) \neq d_{m}$ and thus exerting an active force. In the continuous limit, attached motors exert a continuous force on the filament causing extensional displacement $u(s, t)$. When attached, the motor head moves with a speed that follows a linear force-velocity relationship that varies with the motor extension $y$ as

$$
\partial y / \partial t=-\partial u / \partial t+v_{o}\left(1-k_{m} y / F_{s}\right)
$$

with $v_{o}$ being the maximum (zero-load) velocity of the motor and $F_{s}$ being the stall force. The intrinsic extension $y$ of each motor can take positive as well as negative values.

To connect the force-velocity relationship of an individual attached motor embodied in the equation to the macroscopic deformation of the filament, we consider the biological relevant limits with inter-link (passive links) spacing and inter-motor (active links) spacing much smaller than the filament length. Now when $b \gg \max (w, D)$, a continuum, one-dimensional description of the passively cross-linked, motor-filament aggregate is appropriate. At time instant $t$, we focus on an infinitesimal length $d s$ centered at $s$ of the filament, and average over forces exerted by all motors (attached and detached). The relationship between the mean active force per motor $F_{m}$, the total density of motors $\rho_{m}$ and the averaged (mean) stretch of the motors $\langle y\rangle$ is then given by:

$$
F_{m}=k_{m} N\langle y\rangle,
$$

where the average fraction of attached motors, $N$ satisfies $0 \leq N \leq 1$. In the biologically relevant over-damped limit, inertial effects are not important. Since we ignore bending deformations and retain extensional deformations and motor-generated active forces the strain field $u(s, t)$ satisfies

$$
K_{\mathrm{pas}} \frac{\partial^{2} u}{\partial s^{2}}-G_{\mathrm{pas}} u+\rho_{m} F_{m}=0 .
$$

In equation (11), motor activity is treated as an internally distributed force density per motor. We note that $F_{m}$ and $\langle y\rangle$ are both functions of $s$ and of $t$. In other words, we have replaced the ensemble of active motors (attached and detached) in the element $d s$ centered at $s$ with a mean representative motor that has an average extension $\langle y\rangle$.

In the absence of activity $F_{m}=0$, equation (11) predicts that local perturbations in the extensional strain $u$ decay exponentially with a length scale $\ell_{E} \equiv \sqrt{K_{\text {pas }} / G_{\text {pas }}}=\sqrt{E w / \rho_{\mathrm{N}} k_{\mathrm{N}}}$.

\subsection{Mean field theory for the active force $F_{m}$}

At high motor densities $\rho_{m}$, the number of attached motors is large enough that fluctuations in the time average of the density of attached motors $\rho_{a}(s, t)=\rho_{m} N(s, t)$ are small compared to the mean value. Motor kinetics may then be described using population balances. These flux balance equations relate the attached and detached probability densities $\mathcal{P}_{a}(s, y, t)$ and $\mathcal{P}_{d}(s, y, t)$ to attachment and detachment fluxes $J_{a}$ and $J_{d}$ via attachment (on) and detachment (off) transition rates $v_{\mathrm{on}}(y)$ and $v_{\text {off }}(y)$ respectively. The probabilities satisfy

$$
\begin{aligned}
& \frac{\partial \mathcal{P}_{a}}{\partial t}+\frac{\partial J_{a}}{\partial y}=v_{\mathrm{on}} \mathcal{P}_{d}-v_{\mathrm{off}} \mathcal{P}_{a} \\
& \frac{\partial \mathcal{P}_{d}}{\partial t}+\frac{\partial J_{d}}{\partial y}=v_{\mathrm{off}} \mathcal{P}_{a}-v_{\mathrm{on}} \mathcal{P}_{d}
\end{aligned}
$$


To make analytical progress, we treat the attachment frequency $v_{\text {on }}$ in (12) and (13) as constant. Detachment probabilities are allowed to depend on motor extension $y(s, t), v_{\text {off }}=v_{\text {off }}\left(y, \delta_{m}\right), \delta_{m}$ being a measure of the extension at which the detachment rate becomes significant. We ignore Brownian diffusion of both the motor base and the motor head, so that $J_{a}=(d y / d t) \mathcal{P}_{a}$.

\subsubsection{Equation for ensemble averaged density of attached motors}

We next specify that detached motors rapidly relax to an equilibrium state with mean detached density $\rho_{d}$ and zero motor extension. Simultaneously we assume that the extension of attached motors is sharply peaked about an average extension with transients to this distribution occurring over times very small compared to the macroscopic time scales. Thus $\mathcal{P}_{a}=\rho_{a}(t) \delta(y-\langle y\rangle)$ where $\delta$ here is the Dirac delta function. Treating $y$ as a continuous variable, the mean value of property $X$ is then obtained by integrating over the space of all values of $y$ - i.e, over all stretched configurations of the motors. Formally, we define the average of variable $X$ by $\int X \mathcal{P}_{a} d y \equiv\langle X\rangle N \rho_{m}$, where $\mathcal{P}_{a}$ is the sharply peaked function just introduced. Using the Dirac delta function dependence of $\mathcal{P}_{a}$ to replace the frequency of attachment and detachment $-v_{\text {off }}$ and $v_{\text {off }}$ in equations (12) and (13) with their (ensemble averaged) mean-field attachment rate $\omega_{\text {on }}^{0}$ and the mean field detachment rate, $\left\langle v_{\text {off }}\right\rangle=\omega_{\text {off }}$, we obtain the zeroth moment equation:

$$
\frac{\partial \rho_{a}}{\partial t}=\omega_{\mathrm{on}}^{0} \rho_{m}-\rho_{a}\left(\omega_{\mathrm{on}}^{0}+\left\langle v_{\mathrm{off}}\right\rangle\right)
$$

Here, $\omega_{\text {off }}$ depends on the mean strain $\langle y\rangle$. Following previous work (10) we assume a characteristic motor extension $\delta_{m}$ beyond which detachment is favored. The quantity $\mathcal{E} \equiv k_{m} \delta_{m}^{2} / k_{B} T$ then provides a measure of the energy needed to stretch the motors and cause them to detach. Based on this picture that invokes strain-based (energy based) detachment rate we set $\omega_{\text {off }}=\omega_{\text {off }}^{0} \mathcal{F}(\mathcal{E}, Y)$ with the choice $\mathcal{F}(\mathcal{E}, Y)=\cosh (\mathcal{E} Y)$. Other functional forms yield qualitative results provided $\mathcal{F}$ increases monotonically with $Y$ (see supplementary material ESM Figure 2(a), and subsequent discussions in $§ 3.5$ ).

\subsubsection{Equation for ensemble averaged motor extension or stretch $\langle y\rangle$}

To obtain a closed set of averaged equations, we need an expression for the evolution of the mean dimensionless extension $\langle y\rangle$. This ensemble averaged quantity changes due to three effects - the pre-extension of attaching motors, the fact that motors detach at finite extension and the evolution of the extension whilst the motor is attached. Multiplying (13) by $y$ and then integrating over all $y$ (integrating over orientation space), we find

$$
\frac{\partial\langle y\rangle}{\partial t}=-\left\langle v_{\mathrm{off}} y\right\rangle-\frac{\partial u}{\partial t}+v_{o}\left(1-\frac{k_{m} y}{F_{s}}\right)+\left\langle v_{\mathrm{off}}\right\rangle\langle y\rangle+\omega_{\mathrm{on}}^{0}\left(d_{m}-\langle y\rangle\right)\left(\frac{1-N}{N}\right) .
$$

Using $\left\langle v_{\text {off }} y\right\rangle \approx\left\langle v_{\text {off }}\right\rangle\langle y\rangle$, we obtain

$$
\frac{\partial\langle y\rangle}{\partial t}=-\frac{\partial u}{\partial t}+v_{o}\left(1-\frac{k_{m} y}{F_{s}}\right)+\omega_{\mathrm{on}}^{0}\left(d_{m}-\langle y\rangle\right)\left(\frac{1-N}{N}\right) .
$$

In equation (16), the first term on the right hand side is the stretch due to the passive motion of the attached motor, the second term gives the motor velocity relative to the filament, and the third term (in black) corresponds to the rate at which the mean strain changes due to the kinetics of motor attachment and arises from the difference in extension of attaching and detaching motors.

\subsection{Dimensionless equations and parameters}

We next proceed to simplify (14) and (16) to derive dimensionless forms of these equations and in the process identify important dimensionless parameters. Scaling time with $\left(\omega_{\mathrm{on}}^{0}\right)^{-1}$ and setting $\rho_{a}(s, t)=\rho_{m} N(s, t)$, equation (14) may be recast as

$$
\frac{\partial N}{\partial t}=(1-N)-\Psi \mathcal{F}(\mathcal{E}, Y) N
$$

where the dimensionless number $\Psi \equiv \omega_{\mathrm{off}}^{0} / \omega_{\mathrm{on}}^{0}$ is dependent on the duty ratio of the motors. Defining the dimensionless stretch (extension) $U \equiv u / \delta_{m}$, equation (16) becomes

$$
\frac{\partial Y}{\partial t}=-\frac{\partial U}{\partial t}+\mathcal{A}_{1} \Psi\left(\mathcal{A}_{2}-Y\right)+\left(\mathcal{A}_{3}-Y\right)\left(\frac{1-N}{N}\right) .
$$

In equation (18), we note that changes in $Y$ occur due to three effects - passive convection of motor head when attached, the pre-extension of attaching motors, and corrections due to the fact that motors detach at finite extension. Finally, combining (10) 
Arvind Gopinath and Raghunath Chelakkot and L Mahadevan

\begin{tabular}{|c|c|c|}
\hline Symbol & Definition & Interpretation \\
\hline$G_{\text {pas }}$ & $\rho_{\mathrm{N}} k_{\mathrm{N}}$ & Passive shear modulus \\
$K_{\text {pas }}$ & $E w$ & Extensional modulus \\
$G_{\text {act }}$ & $\rho_{m} k_{m}$ & Active shear modulus \\
$\omega_{\text {on }}$ & & Ensemble averaged motor attachment rate \\
$\omega_{\text {on }}^{0}$ & & Motor attachment rate at zero load \\
$\omega_{\text {off }}$ & & Ensemble averaged motor detachment rate \\
$\omega_{\text {off }}^{0}$ & & Motor detachment rate at zero load \\
$\rho_{a}(s, t)$ & & Density of attached motors at position $s$ \\
$\langle y\rangle(s, t)$ & & Ensemble averaged (mean) motor extension at position $s$ \\
$u(s, t)$ & & Filament elongation (extensional strain) \\
\hline $\mathcal{A}_{1}$ & $v_{o} k_{m} /\left(\omega_{\text {off }}^{o} F_{s}\right)$ & Scaled active velocity \\
$\mathcal{A}_{2}$ & $F_{s} /\left(k_{m} \delta_{m}\right)$ & Scaled active force \\
$\mathcal{A}_{3}$ & $d_{m} / \delta_{m}$ & Scaled active displacement \\
$\mathcal{E}$ & $k_{m} \delta_{m}^{2} / k_{B} T$ & Scaled energy scale for detachment \\
$\Psi$ & $\omega_{\text {off }}^{o} / \omega_{\text {on }}^{o}$ & Scaled duty ratio \\
$N(s, t)$ & $\rho_{a} / \rho_{m}$ & Ensemble averaged fraction of total motors attached \\
$Y(s, t)$ & $\langle y\rangle / d_{m}$ & Ensemble averaged (mean) motor extension \\
$U(s, t)$ & $u(s, t) / d_{m}$ & Scaled filament extension (strain) \\
\hline
\end{tabular}

Table 1: Variables and parameters that appear in the mean-field model. In the first column, symbols in blue indicate dimensional parameters and variables while symbols in black are dimensionless.

and (11) and recognizing that elasticity that multiples the highest derivative of the arc-length will be important in our analysis, we set $\bar{s}=s / \sqrt{K_{\text {pas }} / G_{\text {pas }}}$ to obtain

$$
\frac{\partial^{2} U}{\partial \bar{s}^{2}}-U+\beta N Y=0, \quad \text { where } \quad \beta \equiv G_{\text {act }} / G_{\text {pas }} \quad \text { with } \quad G_{\text {act }}=\rho_{m} k_{m}
$$

Here, $G_{\text {act }}$ is the active analogue of the passive shear modulus. Equations (17)-(19) constitute our mean-field model. We note that the set involves four dimensionless parameters that depend purely on the motor kinetics: (1) $\mathcal{A}_{1} \equiv v_{o} k_{m} /\left(\omega_{\text {off }}^{o} F_{s}\right)$, (2) $\mathcal{A}_{2} \equiv F_{s} /\left(k_{m} \delta_{m}\right)$, (3) $\mathcal{A}_{3} \equiv d_{m} / \delta_{m}$, and (4) $\Psi \equiv \omega_{\text {off }}^{o} / \omega_{\text {on }}^{o}$. Table 1 provides a physical interpretation of these dimensionless parameters. To find steady homogeneous solutions $\left(N_{0}, Y_{0}, U_{0}\right)$ that are independent of both space $\bar{s}$ and time $t$, we set $\partial U / \partial t=\partial Y / \partial t=\partial N / \partial t=0$ in (17)-(19) to obtain

$$
\left(N_{0}, Y_{0}, U_{0}\right)=\left(\frac{1}{1+\Psi \mathcal{F}_{0}}, \quad \frac{\mathcal{A}_{1} \mathcal{A}_{2}+\mathcal{A}_{3} \mathcal{F}_{0}}{\mathcal{A}_{1}+\mathcal{F}_{0}}, \beta N_{0} Y_{0}\right)
$$

where we have defined $\mathcal{F}_{0} \equiv \mathcal{F}\left(\mathcal{E}, Y_{0}\right)$.

Our mean-field approach and analysis leading to equations (17)-(19) complements and differs from previous attempts in a few important ways. First, consistent with experiments suggesting that bond failure is more naturally dependent on the extension and only weakly on the rate of extension, we have chosen $\omega_{\text {off }}^{o}$ to depend on motor extension $(9,10)$ and not the rate of extension (42). Second, non-linear coupling between passive and active deformations in (17)-(19) distinguishes our model from previous studies of motor mediated bending of filaments (42). Finally, our model filament is weakly extensible, bolstered by recent experimental evidence $(3,7,37)$.

Equation (19) ignores passive viscous drag on the deforming filament due to any external fluid resistances. This may be incorporated into equation (11) by adding a term $-\mathcal{A}_{4}(\partial U / \partial t)$ on the left side of the equation proportional to the external viscosity. Supplementary text $§ 1.1$ discusses possible effects of external viscous drag on the active and passive mechanical properties of the substrate. We find that external viscous resistances do not change the qualitative picture of the results discussed below. This is because active attached motors provide both elastic and viscous resistances to local deformation.

\subsection{Persistence length and elasticity in rigor states}

When the motors are in a state of rigor, there is no intrinsic motor dynamics. The motors are stuck in fixed configurations (either attached or detached) and therefore we have $\omega_{\mathrm{on}}^{0}=0, \omega_{\mathrm{off}}^{0}=0$ and $v_{o}=0$. In this limit, the motors are bound permanently to the filament track and can actually sense the instantaneous displacement of the attachment point (where the head is) rather than just the rate of displacement. The number of attached motors $N$ thus cannot change and equals $N_{R}$, the preexisting attached fraction (which may or may not equal $N_{0}$ ). As a result, the motor extension $Y$ responds passively to the change in the attachment 
point of the motor head, so that (18) simplifies to:

$$
\frac{\partial Y}{\partial t}=-\frac{\partial U}{\partial t}
$$

Integrating the above equation once yields

$$
(Y(\bar{s}, t)-Y(\bar{s}, 0))=-(U(\bar{s}, t)-U(\bar{s}, 0)), \quad \bar{s}=s / \sqrt{K_{\mathrm{pas}} / G_{\mathrm{pas}}}
$$

Since $U(\bar{s}, t)-U(\bar{s}, 0)=\delta U$ the filament strain, we have

$$
\frac{\partial^{2} U}{\partial \bar{s}^{2}}-\delta U\left(1+\beta N_{R}\right)=0 .
$$

It is possible that the rigor state is obtained by quenching the motors in configurations where they are not in equilibrium - that is, $N_{R} \neq N_{0}$. Then equation (23) is an equation with non-constant coefficients that in principle can be solved numerically subject to appropriate boundary conditions. Insight may be gained by looking at the case where $N_{R}$ is uniform and then extrapolating it to non-constant functions. When $N_{R}$ is independent of $s$, we matching magnitudes of the two terms and obtain the characteristic length scale $\lambda_{\mathrm{R}}$ over which the strain decays in this rigor state

$$
\lambda_{\mathrm{R}}=\sqrt{\frac{K_{\mathrm{pas}} / G_{\mathrm{pas}}}{\beta N_{R}+1}}=\sqrt{\frac{K_{\mathrm{pas}}}{k_{m} \rho_{m} N_{R}+G_{\mathrm{pas}}}} .
$$

The effective shear modulus due to the links, $G_{\text {eff }}$ is obtained by equating the force needed to shear the composite - here the motor springs act as passive springs. This procedure yields the estimate for the effective modulus

$$
G_{\mathrm{eff}} \sim\left(N_{R} \rho_{m} k_{m}+\rho_{\mathrm{N}} k_{\mathrm{N}}\right) D .
$$

Note that $0 \leq N_{R} \leq 1$ and hence $\lambda_{R}$ s has an upper and lower bound for composite filaments in rigor. A spatially varying $N_{R}$ thus implies that the effective shear modulus and local persistence length may vary with position. Equation (24) is relevant to situations where an initially passive composite is activated. For instance, experiments have shown that flagella starting from a state of rigor may not always spontaneously synchronize and oscillate and instead exhibit localized asynchronous oscillations, especially for very large flagella as in bull sperm. We hypothesize that such behavior can arise when these regions are separated by distances greater than predicted by (24).

\subsection{Analytical predictions for persistence lengths for active assemblies}

The homogeneous and stationary base state, equation (20), is susceptible to perturbations and depending on the values of $N_{0}, Y_{0}, U_{0}, \mathcal{F}_{0}$ and $\mathcal{F}_{0}^{\prime} \equiv(d \mathcal{F} / d Y)_{\left(\mathcal{E}, Y_{0}\right)}$, may be stable or unstable. Furthermore the properties of this base state determine the effective extensional and shear moduli and determine the persistence lengths of strain fields. In the following sections, we will analyze equations (17)-(19) about the base state (20) to derive analytical expressions for the persistence lengths associated with imposed or emergent steady strains, as well as with imposed oscillatory strain fields.

\subsubsection{Emergence of localized strains and localized oscillations}

Before we study the strain field in the full extensible filament, we first examine the stability of the stationary solutions for a small internal section of the composite filament of length much shorter than the relaxation length of pure extensions, i.e. $\ell_{s} \ll \sqrt{K_{\text {pas }} / G_{\text {pas }}}$. In this limit, we can ignore the extensibility of this small section and study local dynamics as it interacts elastically with its neighboring domains. Such a description is also relevant to the dynamics of small local stiff regions of the composite filament starting from a rigor state when $N_{R}$ is spatially varying and the composite is comprised of stiff regions surrounded by relatively soft regions.

The dynamics of this segment relative to its neighbors can be mapped to that of a homogeneous population of motors acting on a rigid segment and working against an external spring with effective stiffness $K_{\mathrm{S}}$ (ESM- $\S$ I and ESM Figures 1 and 2). The spring constant has both active and passive components. The active contribution to this effective spring constant that comes from attached motors in neighboring filaments, and the passive contributions from $k_{N}$ and $E w$. If $\rho_{\mathrm{N}}=0$ then the passive part of $K_{s} \propto K$. Since the fragment is inextensible, the force balance equations (11) and (19) become

$$
-U+\frac{\rho_{m} k_{m}}{K_{s}} N Y=0
$$


(a)

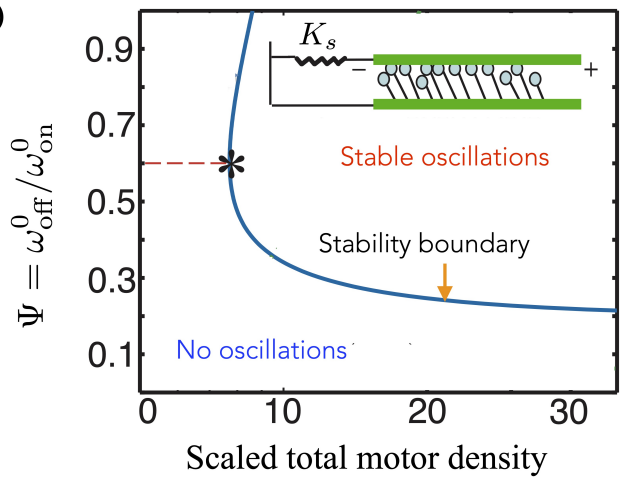

(b)

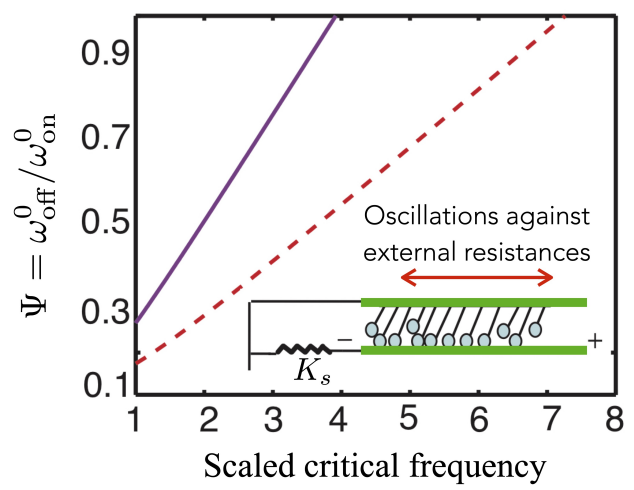

Figure 3: Mean field results for the dynamics of a small rigid fragment working against a resisting spring with $K_{s}>0$ and no external viscosity. Here, $\mathcal{F}=\cosh (\mathcal{E} Y)$. (a) Neutral stability curve $\Psi=\omega_{\text {off }}^{0} / \omega_{\text {on }}^{0}$ vs. scaled density of motors $\rho_{m} k_{m} / K_{s}$ for $\mathcal{E}=4.944, Y_{o}=0.5$ and $\omega_{\mathrm{on}}^{0}=0.25 \mathrm{~s}^{-1}$. For fixed $\Psi$, there is a critical number of motors required for oscillations to manifest (the value for $\Psi=0.6$ is indicated as an example), whereas for fixed motors density $\rho_{m}$, there is a range of $\Psi$ over which oscillations can be sustained. For fixed $\Psi$ there exists a critical motor density, related to $K_{s} /\left(\rho_{m} k_{m}\right)$ above which the actively generated power can balance frictional (chemical) dissipation and enable sustained oscillations. (a) Scaled frequency of oscillations, $\omega_{c} / \omega_{\mathrm{on}}^{0}$ as a function of $\Psi$. The solid line is the exact result while the dashed line is the adiabatic approximation where motor extension is slaved to the filament dynamics (see ESM §1).

For $K_{s}>0$, a globally translating solution ( $U>0$ and constant) is not possible but oscillatory solutions can exist. To discern the existence of such solutions, we analyze the linear stability of the base state (20) to infinitesimal perturbations. Setting $N=N_{0}+N_{1} e^{\sigma t}, Y=Y_{0}+Y_{1} e^{\sigma t}$ and $U=U_{0}+U_{1} e^{\sigma t}$ where $N_{1}, Y_{1}$ and $U_{1}$ are small quantities, we first linearize equations (17)-(19). We then recast the resulting equations in matrix form, evaluate constraints on the matrix for non trivial solutions and study the associated eigenspectrum and variations of growth rate $\sigma$. The eigenvalues thus obtained provide information about the type of instability.

In this case, we find the existence of stable oscillations (limit cycles) for certain values of the parameters. These emergent actively driven oscillatory states bifurcate with well-defined frequencies from the stationary state via a Hopf-Andronov-Poincare bifurcation. In all cases, we find that stable oscillatory solutions exist only within a range of $\Psi$ values. Furthermore, studying the eigenvalues of the linearized equations provides insight into the origin of the oscillations - we find that oscillations are driven by positive feedback - i.e, the emergence of negative effective spring constants and/or negative friction coefficients characterizing the motion of the filament. In turn, these negative coefficients arise to the interplay between the motor kinetics and filament. For large values of $\Psi$, the number of attached motors is insufficient to supply the energy for oscillations thus suggesting an upper bound. For small $\Psi$, too many attached motors increase the net elastic resistance and friction coefficients, causing a strong damping of oscillations. In the stable oscillating state, the active energy input to the system by active motors attaching balances the effective irreversible viscous dissipation due to motor friction as motors detach.

Numerical exploration of the stability boundary is illustrated for a limiting case in Figure 3(a). Figure 3(b) for example plots the correlation between the dimensionless frequency at onset $\omega_{c}$ as a function of $\Psi$ for a particular form of the detachment function with $\mathcal{A}_{1}=0$. More details on the linearization process, analytical expressions for the stability boundary separating stationary and oscillatory states, and for the emergent frequencies may be found in ESM $§ 1$ and ESM Figure 2.

In summary, we have demonstrated that localized steady, or oscillatory extensional strains may spontaneously emerge in a small fragment of a larger active composite. For a filament with infinite elastic modulus $K_{\text {pas }} \rightarrow \infty$, the effect of localized strains is felt everywhere along the filament; this enables spatially separated motor groups to synchronize and behave coherently. Starting from this limit of perfect coherence, we next analyze how elasticity modulates the decay of strains, introduces a persistence length and disrupts coherence. Since we now consider the full composite rather than small interior segments, boundary conditions play an important role in determining the form of the solutions. 


\subsubsection{Persistence of steady deformations in active filament-motor composites}

We begin by identifying the appropriate base state that sets the strain field over the length of the composite filament. The stationary fields $\left(U_{0}, N_{0}, Y_{0}\right)$, here functions of the spatial position $s$, satisfy (19)

$$
\frac{d^{2} U_{0}}{d \bar{s}^{2}}-U_{0}+\beta N_{0} Y_{0}=0
$$

We now choose boundary conditions consistent with the end corresponding to $\bar{s}=0$ held fixed so that $U_{0}(0)=0$. The other end corresponding to $\bar{s}=\ell / \ell_{E}$ is left free and satisfies the force-free condition $\left(d U_{0} / d \bar{s}\right)_{\left(s=\ell / \ell_{E}\right)}=0$. Solving equation (27) with these boundary conditions, we find

$$
U_{0}(\bar{s})=\beta N_{0} Y_{0}\left(1-\alpha e^{\bar{s}}-(1-\alpha) e^{-\bar{s}}\right), \quad \alpha \equiv e^{-2 \ell / \ell_{E}} /\left(1+e^{-2 \ell / \ell_{E}}\right) .
$$

The strain $U_{0}$ is linear in the activity parameter $\beta$ while the decay length is independent of the activity $\beta$. The physical interpretation consistent with the equation is as follows: attached motors sense the strain rates of the filament but they do not have any way to gauge the amplitude (magnitude) strain at the point of attachment at the time of attachment or later. The passive linkers on the other hand sense both the absolute strain as well as strain rates since, unlike active motors, they serve as permanent links. In the absence of any time dependence, the decay length of steady extensional strains can only depend on the passive constituents of elasticity.

\subsubsection{Persistence length of localized oscillatory strains and ability to synchronize}

The final question we address in the context of the continuum model is the decay of non-static, strains in a long filament. Many oscillations in biological contexts have sharply defined frequencies; thus resulting deformation fields may also have well defined frequencies. We therefore next ask how localized oscillatory displacements and equivalently oscillatory extensional strains decay as one moves away from the point of localization (which may for instance coincide with the source of the deformation fields). This question is naturally related to the emergence of coherent dynamics between spatially separated motor domains, each having its own dynamical response. Specifically, this is related to the conditions under which two spatially separated oscillating motor aggregates, lock into synchronous oscillations due to strains transmitted by an elastic connecting filament.

To study this, we need to modify the boundary condition at $s=\ell$ to enable imposed oscillations there, while still respecting the constraints that lead to (28). This is achieved, in asymptotically accurate fashion, by subjecting the free end to a small amplitude oscillatory displacement with frequency $\omega$ and amplitude $\epsilon \bar{U}_{I} \ll \max \left|\bar{U}_{0}\right|$ while making sure that $\epsilon \ll 1$. The last condition ensures that base state that is being probed corresponds to (28) and is both stationary and stable. The boundary conditions then take the form $U(0, t)=0$ and $U\left(\ell / \ell_{E}, t\right)=U_{0}\left(\ell / \ell_{E}\right)+\epsilon U_{\mathrm{I}} \operatorname{Real}\left(e^{i \omega t}\right)$.

Imposing an external frequency such as a localized, oscillatory displacement at the free end will eventually result in the time dependent response of the whole filament with the same frequency. We term this response the frequency-locked and homeostatic response. To probe how strain and activity fields in the filament respond to localized imposed oscillations, we write $(U, N, Y)=\left(U_{0}, N_{0}, Y_{0}\right)+\epsilon e^{i \omega t}(\hat{U}, \hat{N}, \hat{Y})$. We then substitute this form in equation (19) and retain terms to $O(\epsilon)$, to obtain the linear equations

$$
\frac{\partial^{2} \hat{U}}{\partial \bar{s}^{2}}=(1+\beta \bar{\chi}) \hat{U}
$$

where the dimensionless compliance function $\bar{\chi}=-\left(N_{0} \hat{Y}+Y_{0} \hat{N}\right) / \hat{U}$ determines the linear viscoelasticity of the composite filament and depends on motor characteristics such as the stall force $F_{S}$, free velocity, $v_{0}$ and the duty ratio $\Psi$.

Supplementary material section $\$ 2$ examines the form of this function in detail and derives asymptotic expressions in various limiting cases. Examining the susceptibility function in detail, we deduce that the frequency-locked response is possible only when Real $[\chi]$ is negative. The criterion for non-trivial solutions to exist then provides the expression for the effective persistence length scale:

$$
\lambda_{\mathrm{E}}=\sqrt{2} \ell_{E}\left(\sqrt{1+\beta^{2}|\bar{\chi}|^{2}+2 \beta \operatorname{Real}[\bar{\chi}]}+1+\beta \operatorname{Real}[\bar{\chi}]\right)^{-\frac{1}{2}}
$$

with $\beta$ controlling the relative importance of active to passive shear stiffening. In the absence of activity when $\beta=0$, the decay length reduces to its value for a passive filament, that is $\lambda_{\mathrm{E}}=\ell_{E}$. Expansions for weak activity $(\beta \ll 1)$ or for strong activity $(\beta \gg 1)$ are presented in supplementary information $S 2.3$, and their form demonstrates how activity can either enhance or reduce persistence length due to transient shear stiffening provided by the motors. The exact manner of influence depends strongly on the frequency $\omega$ and the ratio $G_{\text {act }} / K_{\text {pas. }}$. For strong activity, $\beta \gg 1, \lambda_{\mathrm{E}} \approx \sqrt{2\left(K_{\mathrm{pas}} / G_{\mathrm{act}}\right)}(\mathcal{R}+|\chi|)^{-\frac{1}{2}}$ with actively driven shear stiffening dominating. Equation (30) implies that the persistence length is influenced by the imposed frequency $\omega$, 
(a)
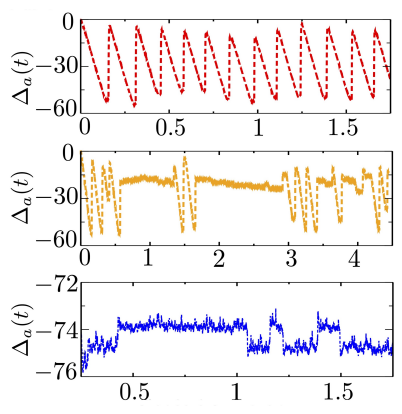

Scaled simulation time

No oscillations

Intermittent oscillations

- Regular oscillations (b)

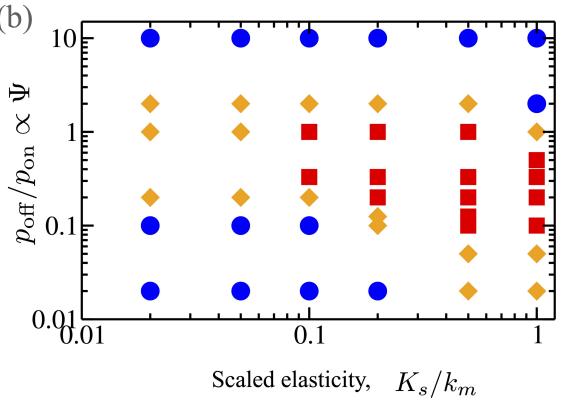

(c)

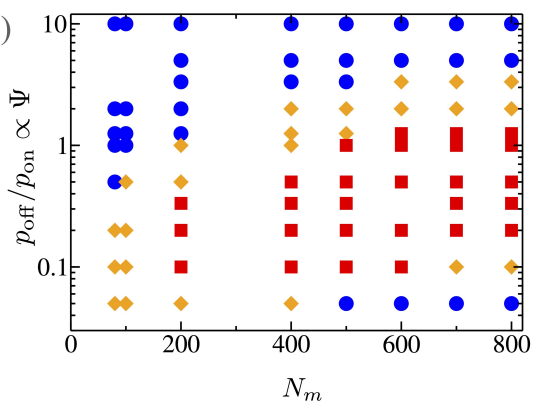

(d)

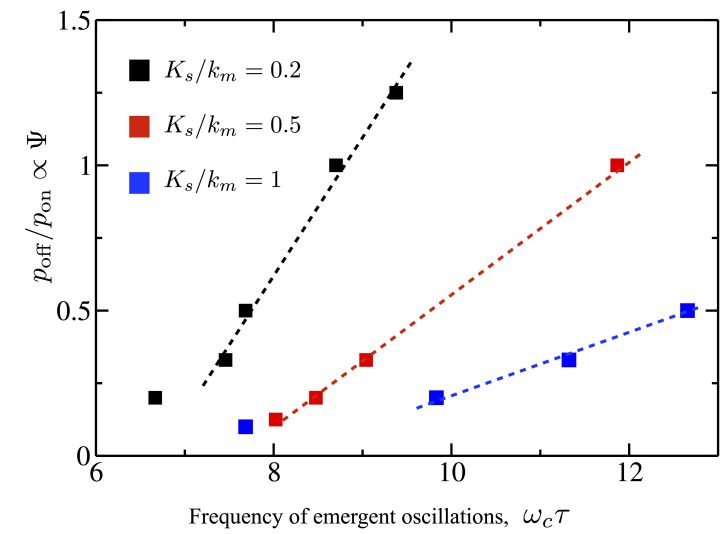

Figure 4: (a) The time dependent displacement of the free end, $\Delta_{a}(t) / \sigma$ for a fixed value $K_{s} / k_{m}=0.2$, and for zero load motor detachment/attachment probabilities, $p_{\text {off }} / p_{\text {on }}=1.0$ (top), 2 (middle) and 10 (bottom), illustrating various dynamical regimes observed in simulations. Phase diagram from the Brownian-MPC simulations indicating the dynamical regimes as a function of (b) the number (density) of total motors acting on the segment and (c) of the ratio $K_{s} / k_{m}$. The plots show how the stability boundary is influenced by $p_{\text {off }} / p_{\text {on }}$. The observed dynamical regimes are, steady extension (blue, circle), intermittent oscillations (orange, diamonds), and steady oscillations (red, squares). Colored regions are a guide to the eye. (d) The oscillation frequency of a periodically oscillating filament also obtained from the Brownian-MPC simulations with $K_{s} / k_{m}=0.5$.

though the frequency dependent value of $\bar{\chi}$. In the low frequency limit, motors undergo multiple mechanochemical cycles before they feel the imposed oscillation. In the high frequency limit, attached motors encounter several cycles of imposed oscillations before detaching. These trends are transparent when we examine the limit $\mathcal{A}_{1}=0$. Specifically, in the high frequency limit activity increases the persistence length from the lower bound $\lambda_{\mathrm{E}} \rightarrow \ell_{E} / \sqrt{1+\beta}$ as $\omega \rightarrow \infty$.

\section{BROWNIAN-MPC SIMULATIONS OF NOISY COMPOSITE FILAMENTS}

Having studied the role of shear stiffness due to passive links in controlling the bending and extension of deformed filaments, we now describe our discrete, microscopic model that includes noise due to motor activity. This allows us to achieve two objectives. First, we can qualitatively compare the influence of noise in systems containing filaments and motors by selecting a finite number of motors and second, we can adjust the discrete version of the filament elasticity $K_{\text {pas }}$ and study stiff, moderate as well as soft filaments. The latter two regimes are inaccessible to the mean-field theory.

\subsection{Numerical model}

The discrete analog of the weakly extensible filament is modeled as a one dimensional chain of $M$ rigid segments (the unit cell is illustrated in Figure 3(a) and the complete composite filament in Figure 5(a)). Each of these segments has length $\ell_{s}=80 \sigma$ and is made up of $M_{m}$ discrete spherical monomers of diameter $\sigma$ located at $r_{i},\left(i=1, \ldots, N_{m}\right)$. The monomers are connected by an elastic potential that controls the stretchability within each segment

$$
u_{s}=\frac{\kappa_{s}}{2} \sum_{i=1}^{M_{m}-1}\left(\left|\mathbf{r}_{i+1}-\mathbf{r}_{i}\right|-\sigma\right)^{2}
$$


(a)

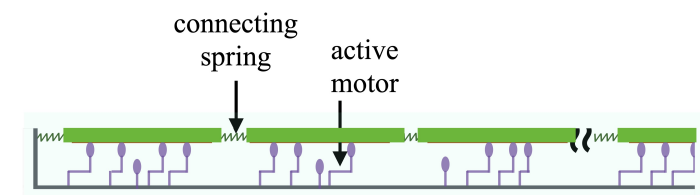

Tethered

end

Effective elasticity and persistence of strain in active filament-motor assemblies

(b)

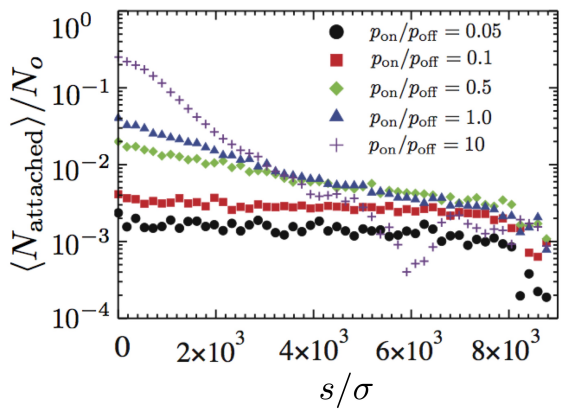

(c)

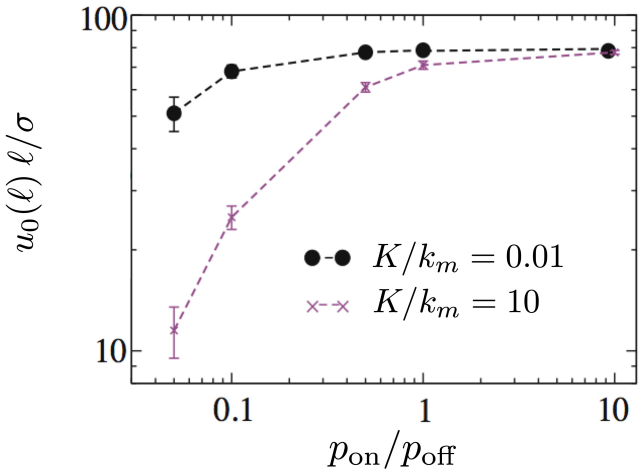

Figure 5: (a) Sketch of a discrete, active composite filament we analyze in this paper. The composite filament is made of 50 similar rigid segments each connected to its neighbors by a linear elastic spring with spring constant $K$. One end of the segment (Index 1) is anchored to a wall by a similar spring while the other (free) end (Index 50) is oscillated with a frequency $\omega$. Each segment interacts with 80 active motors that periodically attach and detach from the filament. (b) The fraction of motors attached on the composite filament $\left\langle N_{a}\right\rangle$, as a function of their position from the clamped end for $K / k_{m}=10$. The mean motor attachment is maximum close to the clamped end, where the local strain is relatively small. (c) Time averaged steady state extension of the segment at the free end, $\left\langle u_{0}(\ell)\right\rangle$, as a function of zero load motor attachment/detachment probability, $p_{\text {on }} / p_{\text {off }}$, for various values of passive spring stiffness, $K / k_{m}$. The extension increases with $p_{\text {on }} / p_{\text {off }}$, and decreases with $K / k_{m}$, consistent with analytical predictions. For large $p_{\text {on }} / p_{\text {off }}$ the extension saturates at a maximum value, set by the finite length of the filament-motor composite.

Each segment is also connected to neighboring segments by linear elastic spring of stiffness $\propto K_{\text {pas }}$ (the proportionality constant being dependent on $\sigma / \ell$ ) with rest length $80 \sigma$. With $\ell \gg \ell_{s}$ since $M \gg 1$, bending and extensional deformations can be ignored within each segment. The first segment is connected to a rigid wall by the same elastic potential at location corresponding to $s=0$ while the other end of the composite at $s=\ell$ is kept free. Bending stiffness is implemented via a three-body bending potential

$$
u_{\mathrm{b}}=\frac{\kappa}{2 \sigma} \sum_{i=2}^{M_{m}-1}\left(\mathbf{t}_{i+1}-\mathbf{t}_{i}\right)^{2}
$$

where $\mathbf{t}_{i}=\left(\mathbf{r}_{i}-\mathbf{r}_{i-1}\right) /\left|\mathbf{r}_{i}-\mathbf{r}_{i-1}\right|$. The effective bending rigidity $\kappa$ penalizes angular changes from a local straight geometry for the segments. In our simulations $\kappa_{s}=2 \times 10^{4}\left(k_{B} T / \sigma^{2}\right)$ and $k / \sigma=2 \times 10^{4}\left(k_{B} T\right)$ with an appropriately large persistence length for the segment $\sim 250 \ell_{s}$. While our model is general enough to include bending deformations by treating $\kappa$ as a variable parameter - here we focus solely on extensional strain on the filament.

Motors that impose active forces that cause filament shear and extension are modeled as linear elastic springs with stiffness $k_{m}$ and rest length $\ell_{m}$. These motors are uniformly distributed on each segment with number density $\rho_{m}$. One end of the motor (tail) is grafted to a point $\mathbf{r}_{t}$ on a rigid base while the other end (head) can attach and detach to a given monomer, located at $\mathbf{r}_{h}$, of the filament. During the attachment process, the monomer bead which lies closest to the motor head is chosen so that the motor extension, $\left|\mathbf{r}_{h}-\mathbf{r}_{t}\right|-\ell_{m}$, upon attachment is minimal. Similar to the mean-field model, we assume a constant attachment probability $p_{\text {on }}$ for each motors. Once attached, the unidirectional motor head steps to the next monomer in the direction of the anchored end, $s=0$, with a discrete step size that equals the monomer diameter $a$. As the motor heads move, the accompanying extension of the motor length leads to a force $\mathbf{F}_{m}=-\kappa_{m}\left(\left|\mathbf{r}_{h}-\mathbf{r}_{t}\right|-\ell_{m}\right) \hat{\mathbf{e}}$, where the unit vector $\hat{\mathbf{e}}=\left(\mathbf{r}_{h}-\mathbf{r}_{t}\right) /\left|\mathbf{r}_{h}-\mathbf{r}_{t}\right|$, that acts on the attached filament. This force is balanced by the tension on the filament to which the motor is attached, thus resulting in a localized active strain on the filament. We assume a load dependent stepping velocity of the motor head, choosing a linear relationship, $v_{h}=v_{o}\left(1-\left|\mathbf{F}_{m}\right| / f_{\max }\right)$ with $f_{\max }$ being the stall force $F_{s}$ consistent with the form assumed in the mean-field theory. Motor heads that are attached to the filament sense the load and detach at a rate given by the load dependent probability 
(a)

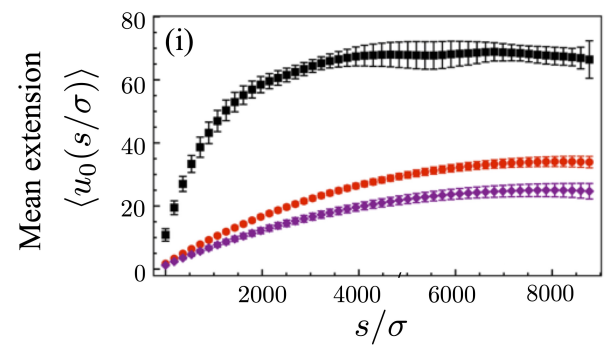

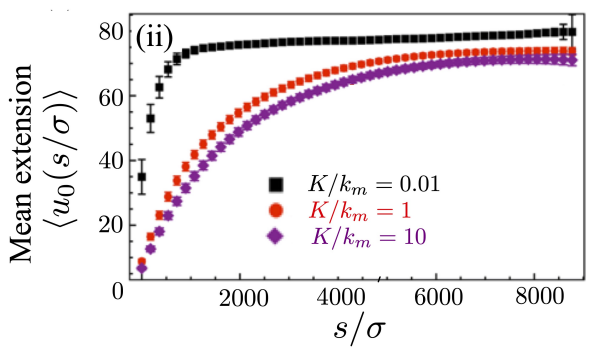

(b)

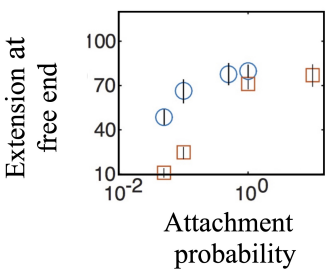

Figure 6: (a) The dimensionless mean steady state extension of material points $\left\langle u_{0}(s)\right\rangle \ell / a$ as a function of their position from the clamped end $s=0$ for three values of $p_{\text {on }} / p_{\text {off }}$ - (i) 0.1 and (ii) 1.0. Vertical bars correspond to the root mean square deviation and indicate the extent noise plays in disturbing the mean value. Other parameters correspond to Figure 5. (b) The extension at the free end as a function of attachment probability $p_{\text {on }}$ for soft $\left(K / k_{m}=0.01\right.$, circles, online-blue) and stiff $\left(K / k_{m}=10\right.$, squares, online-red) filaments.

$\tilde{p}_{\text {off }}$, defined by the piecewise function

$$
\tilde{p}_{\text {off }}= \begin{cases}p_{\text {off }}, & \text { if }\left|\mathbf{F}_{m}\right| \leq f_{\text {cut }} \\ 1, & \text { if }\left|\mathbf{F}_{m}\right|>f_{\text {cut }},\end{cases}
$$

where $p_{\text {off }}$ is the load independent detachment rate of motors and $f_{\text {cut }}$ is the motor cut-off load. We take $f_{\text {cut }}=f_{\text {max }}$ in our simulations, although in general they are not necessarily equal.

The load dependence of the detachment probability of individual motors allows for the crucial two-way coupling between filament elasticity and the motor response and also sets the critical motor extension at which detachment occurs. Furthermore, the constant load independent detachment probability $p_{\text {off }}$ in conjunction with $p_{\text {on }}$ yields an equilibrium fraction of attached motors, $p_{\text {on }} /\left(p_{\text {on }}+p_{\text {off }}\right)$. We recall that in the continuum theory, the steady state fraction of attached motors is $\rho_{a} / \rho_{m}=\omega_{\text {on }}^{0}\left(\omega_{\text {on }}^{0}+\omega_{\text {off }}\right)^{-1}$. In the absence of any mean motor deformation (ie., when $Y_{0}=0$ so that $\left.\mathcal{F}=1\right)$, this fraction equals $\omega_{\text {on }}^{0}\left(\omega_{\text {on }}^{0}+\omega_{\text {off }}^{0}\right)^{-1}$ providing the mapping between the frequency of attachment/detachment in the continuum theory and probabilities of attachment and detachment in the stochastic simulation. However, we note that despite being a discretized model, the segments in the filament still represent a coarse grained description of the filament in biological systems and hence we allow multiple motor heads to occupy the same site on the filament. Because of the initial distribution of the motors, the torque on the filament due to motor activity is negligible and the system is essentially one dimensional.

For simplicity and to focus on the role of activity and noise, we did not incorporate permanent passive cross-linkers of the mean-field model. This the simulation corresponds to the limiting case $\rho_{N}=0$. This does not change the qualitative features of the solution since, shear resistance is always provided by active motors when attached. With this simplification, the passive elasticity of the filament is contributed solely by the weak extensional elasticity of the filament. We point out that the simulation is truly one dimensional since we simulate a bead-spring filament of thickness $a$ rather than a quasi-1D strip of width $b \gg w$ considered in the theory.

The time dependent evolution of this system of motors and segments are analyzed in the over-damped limit using Brownian Multi-particle Collision Dynamics (Brownian-MPC) method (43). This method neglects long range hydrodynamic interactions between the segments while keeping the system in a thermal bath. According to this scheme, each monomer beads of the segment independently performs a stochastic collision with phantom fluid particles with momenta taken from the Maxwell-Boltzmann distribution with variance $\rho k_{b} T, \rho$ being the phantom fluid density. In between these collision events, the monomer positions velocities are updated using standard molecular dynamics (MD) scheme. The timescale for Brownian-MPC simulation $t_{\mathrm{MPC}}=\sqrt{m \sigma^{2} / k_{B} T}$, where $m$ is the mass of a phantom-fluid particle, $a$ is the diameter of the monomer bead which equals the length-scale arises from the discretization of space in the simulation scheme (43). However, the physical timescale relevant to this problem is the numerically calculated thermal relaxation time of the segment of length $80 a$, which is much larger than $t_{\text {MPC. }}$. This relaxation time is determined by the viscosity of the medium set by the simulation parameters $(43,44)$. For the same set of fluid parameters used in reference (44), we find the relaxation time, $\tau$, of the segment of length $80 \sigma$ to be about $4.6 \times 10^{3} t_{\mathrm{MPC}}$. 
Effective elasticity and persistence of strain in active filament-motor assemblies
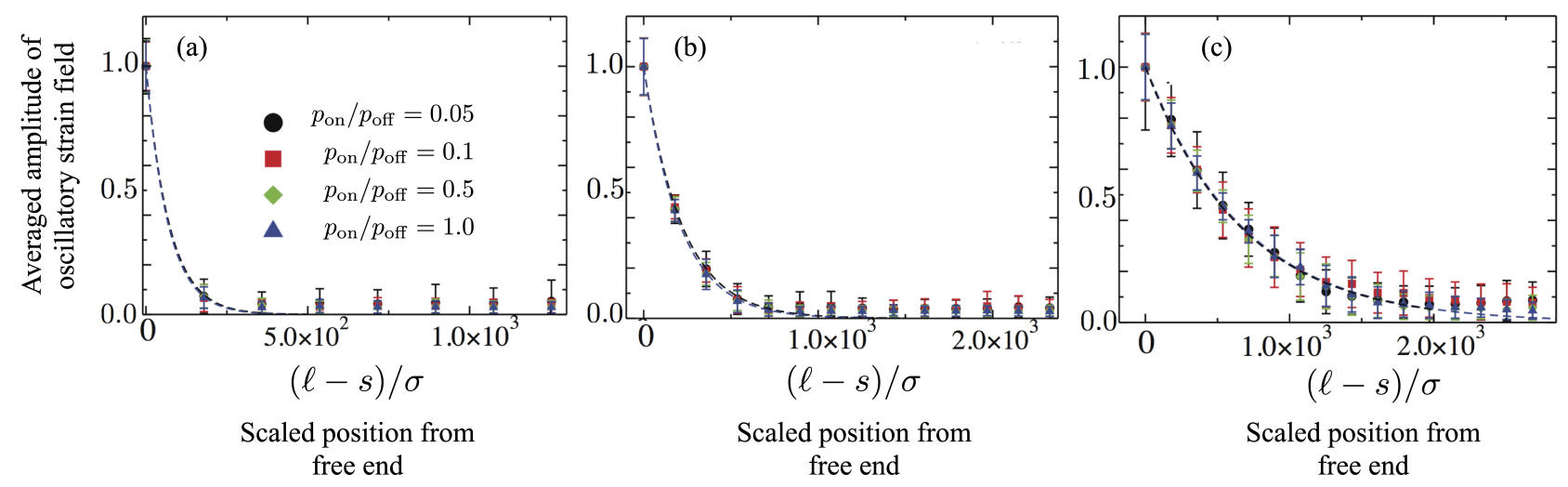

Figure 7: (a)-(c) The noise averaged amplitude of spatial oscillatory field of the filaments as a function of position $s$, for different values of the stiffness parameter - i.e., the elasticity contrast (a) $K / k_{m}=10^{-3}$, (b) $10^{-2}$ and (c) $10^{-1}$. Here $\ell$ is the total length of the composite filament - we note this allows us to define an average coarse-grained elastic modulus per length. The dashed curves show exponential fits indicating decay lengths whose value depends on motor kinetics via the ratio of attachment to detachment probabilities as well as on the elasticity contrast. The (dimensionless) forcing frequency is $10^{-3} v_{0} / \sigma$.

\subsection{Identifying a suitable stationary stable deformed state}

As in our mean field theory §3.5.1 and §3.5.2, we first study the dynamics of a single, rigid fragment, here a single segment of length $\ell_{s}=80 \sigma$, driven by an aggregate (75-800 in number thus corresponding to different motor densities) of active motors. The arrangement is as shown in the inset in Figure 2; as there, the one end of the fragment is attached to an anchored linear spring. For this simple case we choose the spring constant to be $K_{s}$. The other end is free. The spring has, without loss of generality, a zero rest length. We emphasize that in the stochastic model, the passive shear springs are not incorporated. Motor activity results in the sliding motion of this rigid segment. This motion is coupled to the deformation of the anchoring spring. The dynamical response of this system is studied as a function of two parameters, a stiffness parameter $K_{s} / k_{m}$ and the activity parameter $p_{\text {off }} / p_{\text {on }}$. This is done by tracking the displacement of the segment as a function of time as well as the temporal variations in the fraction of active motors attached to the segment. Times are scaled using $\tau \simeq 4.6 \times 10^{3} t_{\mathrm{MPC}}$. This simulation allows us to characterize the dynamical regimes of that segment as a function of motor activity and elastic stiffness. More importantly, this allows us to identify the appropriate base state to use in our calculation of the decay lengths of imposed or inherent local extensional strains.

Depending on the values of $K_{s} / k_{m}$ and $p_{\text {off }} / p_{\text {on }}$ we find three distinct dynamical regimes as illustrated in Fig. 4(a). For a fixed value of $K_{s} / k_{m}$ when $p_{\text {off }} / p_{\text {on }} \ll 1$ a majority of the motors are attached to the filament, subjecting the segment to a significant active force. Consequently, the segment displays a steady extension. In the complementary limit, where $p_{\text {off }} / p_{\text {on }} \gg 1$ the re-attachment rate of the motors is small, causing a majority of the motors to be in the detached state. Thus, in the absence of any significant extensional force the filament attains a steady, but non-extended state. However there is a third time dependent regime observed for intermediate values of $p_{\text {off }} / p_{\text {on }}$; in this regime, we observe intermittent (Fig. 4(a)-middle) or regular (Fig. 4(a)-top) oscillations of the segment with concomitant extensions and compressions of the linear spring. These oscillations are quantified by measuring the distance of the free end of the filament from the anchor point $\left(\Delta_{a}\right)$ as a function of time. The stable, regular oscillatory state is marked by a well defined frequency while in the intermittent oscillatory state, the segment randomly switches between stationary and oscillatory states.

Figure 4(b) explores the complementary phase space where the number of motors is kept fixed (at 800) while the ratio $K_{s} / k_{m}$ is varied. We observe that oscillations for fixed motor density can be triggered or suppressed by tuning the ratio $K_{s} / k_{m}$. The striking difference between the theoretical predictions and the simulation results is the presence of a intermittently oscillating state (yellow, diamonds). A sweep of the parameter space yields the computational phase plot in Figure 4(c) that maps the regimes in the space spanned by the ratio $p_{\text {off }} / p_{\text {on }}$ and the number of motors. This plot is qualitatively similar to and consistent with the theoretical predicted one shown in Figure 3(b). Recognizing the equivalence of $\Psi$ and the ratio $p_{\text {off }} / p_{\text {on }}$, we note that for a fixed number of total motors equivalent to a fixed stiffness parameter oscillations (red, squares) are observed only for a range of $\Psi$. In the absence of noise, the boundary in parameter space between stationary and oscillatory states is sharp. Discrete noise however smears this sharp boundary and renders it fuzzy. Thus the intermittent states are sustained by 
noise - here due to the discrete motor binding and unbinding events and due to the finite number of motors. We emphasize that there is no thermal noise in the system and anticipate that the presence of thermal noise will probably broaden the intermittent region further. Focussing on the regular oscillatory regime, we also compute the frequency as a function of the $p_{\text {off }} / p_{\text {on }}$ at fixed stiffness ratio as shown in Fig. 4(d) and find that the response is consistent with the mean-field predictions, with frequency of oscillations $\omega_{a}$ increasing with the ratio $p_{\text {off }} / p_{\text {on }}$. This may be compared to Figure 2(b) where we found that oscillation frequency was predicted to increase almost linearly with increasing values of $\Psi$.

The physical picture underlying the onset of these oscillations may be understood as follows. The motion of the filament due to motor activity results in a non-linear viscoelastic coupling between the motors, the spring and the irreversible motor friction. At criticality, the motion is determined by the linear response to small perturbations. In this limit, the viscoelastic coupling may be reduced to effective, linear, in-phase (effective elastic) as well as out of phase (viscous) compliance terms. Oscillations arise when either of these terms turn negative as a result of positive feedback.

In a similar vein, it is possible to qualitatively understand how the frequency of emergent oscillations depends on the motor kinetics. When the system is in the stable oscillatory state, the power input to the system due to motor activity balances the energy dissipated due to frictional damping caused by motor attachment. Invoking expressions from the continuum theory presented earlier, we estimate the active friction due to motors to be $\eta_{\text {act }} \sim \rho_{m} N_{0} k_{m}\left(d\langle y\rangle / d u_{t}\right)_{u_{t}=0}$ where $u_{t}=d u / d t$ and $\rho_{m} N_{0}$ is the total number of attached motors. As $\Psi$ increases, both $N_{0}$ and $\left(d\langle y\rangle / d u_{t}\right)_{u_{t}=0}$ are expected to typically decrease, and therefore the frequency of the emergent oscillations should increase. This prediction is consistent with the simulation results in Figure 4(d).

\subsection{Persistence of steady deformations in noisy active filament-motor composites}

Having identified the dynamical regimes of a single rigid segment, we now analyze the dynamical behavior of a long, extensible composite filament as sketched in Fig. 5(a). This composite filament is made up of 50 rigid segments, each of length $80 \sigma$. The anchored end is chosen to correspond to $s=0$ while the free end corresponds to $s=\ell$. The variable $s$ here thus measures distance from the anchored end and is identical to the arc-length parameter introduced in the continuum model.

As before, the overall global softness of the filament is controlled by the elastic constant of connecting springs, here denoted by $K$ and proportional to the modulus $K_{\text {pas }}$ in the continuum mean-field theory. We distribute 80 motors uniformly on each of the segments and choose simulation parameters such that the dynamical state of each of the segments corresponds to steady, non-oscillatory extension, $u_{0}(s)$. The choice of the parameters is guided by the results for the unit cell (single segment) case. The base state chosen was confirmed to be stationary so that each segment as well the the whole composite filament is free of oscillatory instabilities. Due to the stochastic nature of the motor dynamics, all the steady quantities have a fluctuating component. We measure the mean of these quantities by taking a time average after the system has evolved into a steady state.

Since the motor kinetics is coupled to the inhomogeneous filament strain, the motor imposed filament extensions modifies the number of attached motors on the filament. In Fig. 5(b) we plot the mean fraction of motors attached, $\left\langle N_{a}\right\rangle$, as a function of $\mathrm{t} s$ for $K / k_{m}=10$ and for different values of $p_{\text {on }} / p_{\text {off }}$. When $p_{\text {on }} / p_{\text {off }}<0.1$ the motor imposed extension of the filament is weak as the number of attached motors is small and the motor distribution is roughly uniform along the filament. However when $p_{\text {on }} / p_{\text {off }}>1$ the enhanced motor attachment imposes significant extension to the filament, which is maximum at the free end. The nonuniform extension of the composite leads to a nonuniform distribution of attached motors with the mean motor attachment is maximum near the anchored end $(s=0)$, where the filament extension is minimum as seen in Fig. 5(c). The attached fraction decreases with the distance from that point as the filament strain increases as one moves towards the free end.

The time averaged steady motor induced extension $\left\langle u_{0}(s)\right\rangle$ of the filament as a function of the stiffness parameter $K / k_{m}$ are plotted in Fig. 6 (a)-(i) \& (ii) for two values of $p_{\text {on }} / p_{\text {off }}$. Note that $p_{\text {off }}$ is constant in these plots; the ratio is changed by varying just the detachment probability. As seen already in Fig. 5(c), filament extension is minimum near the clamped end and maximum at the free end; furthermore both $p_{\text {on }} / p_{\text {off }}$ as well as $K / k_{m}$ equally influence the value as well as the gradient in the extensional strain. In general, $u_{0}$ increases with the fraction of attached motors $\left(\propto\left(p_{\text {off }} / p_{\text {on }}\right)^{-1}\right)$ and filament softness $\propto\left(K / k_{m}\right)^{-1}$. In continuum limit, as anticipated by the mean-field theory (see ESM $\S 3$ ) the steady state extension $\left(u_{0}\right)$ in the limit of no permanent cross-linkers is

$$
u_{0}(s) \propto\left(\frac{\rho_{m} k_{m}}{2 K}\right) s(2 \ell-s)
$$

where the constant of proportionality depends purely on motor kinetics and is independent of position. Note that this form is also evident from equation (30) where motor activity and kinetics appears as a prefactor multiplying the function that encodes the spatial variation. In our simulation, we do not have passive linkers and this $\rho_{N}=0$. Therefore, $u_{0}(s)$ does not have any have a characteristic decay length; this is because attached active motors form only temporary attachments and do not contribute to the long time filament (shear) elasticity. The discrete nature of motor activity causes large fluctuations in mean extension of the filament, especially for very soft filaments with $K / k_{m} \ll 1$ (as will also be discussed in see Fig. 8(a,b)). We also find 
(a)

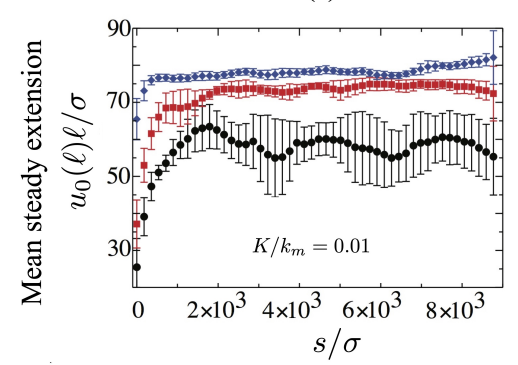

Effective elasticity and persistence of strain in active filament-motor assemblies

(b)

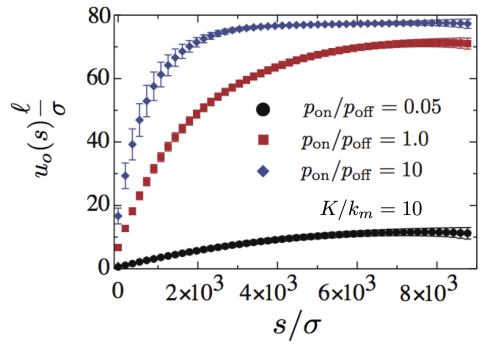

(c)

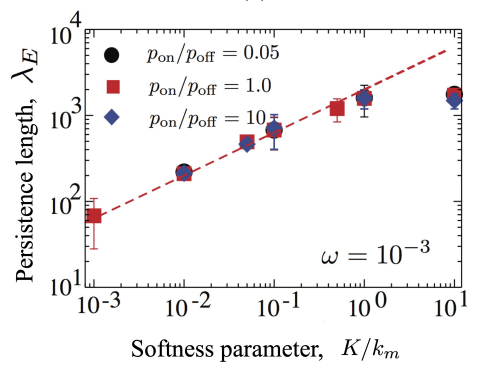

Figure 8: Summary of our results (a,b) Scaled mean steady extension as a function of position from the clamped end. Vertical bars correspond to the RMS deviation. Extensions are observed to be uncorrelated for soft assemblies $\left(K / k_{m}=0.01\right)$ with domains fluctuating independently. Increasing stiffness allows separated parts of the filament to move more in consort. Note that changing the motor kinetics also affects the size of these independent domains. The strain gradient localizes close to the clamped end for soft assemblies, especially for large attachment probabilities. We expect large fluctuations (induced or arising intrinsically) to have more of an effect in disrupting coherent behavior in these domains. (c) Decay lengths $\lambda_{E}$ obtained by analyzing the amplitude of oscillations as a function of $K / k_{m}$ for various $p_{\text {on }} / p_{\text {off }}$. For small values of the ratio (weak elasticity) the simulations agree with the theoretical scaling predictions, $\lambda_{E} \propto \sqrt{2 K / k_{m} \rho_{m}}$ shown as the dashed line. We see evidence of system size saturation for large values of $K / k_{m}$ and hence the deviation from the mean-field prediction. The (dimensionless) forcing frequency of the oscillation at the end is $10^{-3} v_{0} / \sigma$. Results are qualitatively similar for small forcing frequencies. As the frequency increases, the frequency-locked response may not be a valid assumption and the concept of a decay length loses its significance.

from additional simulations that motor noise results in uncorrelated spatial domains for $K / k_{m}=0.01$ while a greater degree of correlation is observed for $K / k_{m}=10$.

The Brownian-MPC simulations are an improvement over the mean-field theory in an important way; in the theory, the local attachment fraction was spatially invariant and independent of position along the filament. In our case, the coupling between elasticity and motor kinetics is intrinsic and non-linear. A consequence of this is the strong variation of the attached motor fraction with position from the clamped end evident in Figure 5(b) for high attachment probabilities. Note also that the quadratic nature of extension field, predicted from mean-field theory is qualitatively consistent with the numerical results. Allowing for the attached motor fraction to vary with position allows one to incorporate some non-linear coupling effects and predict the deviation from the quadratic scaling of the extensional strains closed to the clamped end.

\subsection{Decay lengths of oscillatory strains}

To study the decay of localized oscillatory strains in this noisy, active setting we simulate the model problem analyzed in Section 3.5.3. We impose a low frequency $\left(\omega \ll v_{0} / \sigma\right)$, small amplitude $\left(u_{1} \ll u_{0}(\ell)\right)$ oscillation at the free end $(s=\ell)$ about the steady stationary extension that is attained in the absence of this imposed deformation. Thus, the net extension imposed (and measured) at the free end is $u(\ell)=u_{0}(\ell)+u_{1} \sin (\omega t)$. Since the motors do not form permanent cross-links, this steady state deformation is identical to the deformation attained as the frequency goes to zero. The oscillation at $s=\ell$ results in a spatially and temporally varying extensional field $u(s)$ along the contour of the filament. At long times, after initial transients have died out, this field may be written as the sum of the actively generated steady strain $u_{0}$ and an oscillatory strain of amplitude $\hat{u}(s) \propto u_{1}$. For small deformations, the ratio $\hat{u}(s) / u_{1}$ depends only on $s$, the filament elasticity and motor kinetics. In the frequency-locked limit $\hat{u}(s) / u_{1}$ oscillates with frequency $\omega$ - the critical assumption here is that intrinsic oscillatory instabilities are not excited due to motor activity.

Focusing on the amplitude $\hat{u}$ of the oscillatory part of the strain, we compute the spatial variation and fluctuations (averaged over many motor cycles) in the amplitude for each segment as a function of its distance from the free end $(\ell-s)$. For sufficiently low frequencies, the results are qualitatively independent of the frequency $\omega$ as the system is truly frequency-locked. For high frequencies, we observed that in some cases localized oscillations were initiated - for these systems the notion of a persistence length breaks down and is not a useful physical measure. Hence we will focus on the low-frequency results and in particular the value $\omega=10^{-3}\left(\sigma / v_{0}\right)$. In the case of an inextensible filament, the oscillatory strain that is imposed at the free end would propagate an infinitely long distance without any decay in its amplitude. However, for finitely extensible filaments, equation (29) 
predicts an exponential decay of strain along the filament. Indeed, simulations confirm this feature. Figures 7(a)-(c) show an exponential decay for the scaled amplitude of the oscillatory extension $\hat{u} / u_{1}$ as a function of distance from the free end for different values of $K / k_{m}$ and $p_{\text {on }} / p_{\text {off. }}$. The amplitude $\hat{u}$ does show large fluctuations due to the noise imposed by discrete motor attachment/detachment - something the mean-field theory cannot capture. The length scale over which the elongation decays $\lambda_{E}$ is seen to be a strong function of $K / k_{m}$, but surprisingly, only a weak function of $p_{\text {on }} / p_{\text {off }}$ in the regime we probed. We hypothesize that this is because the system has access to the dynamical parameter space where the average number of attached motors is small and the contribution to the elastic stiffness due to motor attachment is negligible.

In summary, we find that the decay of steady, motor-mediated, localized extensions is controlled by $K / k_{m}$ and the ratio of detachment to attachment probabilities, $p_{\text {off }} / p_{\text {on }}$, for both stiff $\left(K / k_{m}=10\right)$ and soft $\left(K / k_{m}=10^{-2}\right)$ filaments. The mean field prediction is qualitatively consistent with the simulation results for $p_{\text {off }} / p_{\text {on }} \sim 1$ with deviations seen for large contrasts between the probabilities. Increasing the attachment probability results in a larger fraction of attached motors with sharper gradients near the clamped end and flatter profiles near the free end consistent with analytical predictions. Motor noise results in large fluctuations in the mean extension, noticeable especially for the soft filaments and also results in uncorrelated spatial domains in extension as demonstrated in Figures 8(a) and 8(b). For a fixed $\omega$, we estimate the decay length by fitting $\hat{u}(\ell-s)$ to an exponential function for a range of $K / k_{m}$ and $p_{\text {on }} / p_{\text {off. }}$. Our calculations show an increase in $\lambda_{E}$ as $K / k_{m}$ is varied from $10^{-3}$ to 10 . We plot the computationally calculated $\lambda_{E}$ for the noisy system as a function of $K / k_{m}$ in Fig. 8(c). The analytical prediction (30) may be simplified for our system with no permanent linkers by taking the limit $G=0$. Making this change and recasting the resulting expression we get

$$
\lambda_{E}=\left(\frac{2 K_{\mathrm{pas}}}{G_{\mathrm{act}}}\right)^{\frac{1}{2}}\left[\frac{1}{\sqrt{|\bar{\chi}|+\operatorname{Real}(\bar{\chi})}}\right] \propto \sqrt{\frac{2 K}{k_{m} \rho_{m}}} .
$$

Our simulation results shows excellent agreement with this prediction for almost all of the range explored in $K / k_{m}$. For very stiff filaments, we find that the computational value deviates from the dashed line and seems to saturate to a constant. We ascribe this behavior to finite system size effects. We also computed the decay length for imposed frequencies (dimensionless) $\omega=10^{-2}$ and found qualitatively similar results. For instance, when $K / k_{m}=1$, the decay length is proportionally smaller than for $\omega=10^{-3}$ due to the dependence of $\chi$ on the frequency.

\section{CONCLUSION}

The scale over which active deformations persist in a fluctuating environment is a fundamental question that arises in the study of living, active and activated matter. Here, this question is addressed in the context of an ordered composite structure consisting of elastic elements that can be stretched and sheared by active motors binding to and unbinding from them. We present a mean-free theory in the weak noise and weakly elastic limit as well as detailed Brownian multi-particle collision dynamics-based numerical simulations for noisy, moderately soft and soft composites. Both theory as well as simulations show that extensibility may be negligible locally but it cannot be ignored globally. This is because the length scale over which deformations persist is controlled by the competition between filament extensibility and shear stiffening conferred by the permanent passive cross links as well as temporary attachments formed by the active motors.

In nearly stiff filaments for which extensional stains are negligible, the interplay between activity and shear elasticity can result in stable stationary or oscillatory extensions. Motor noise due to discrete binding and unbinding events and due to finite motor numbers results in a fuzzy boundary exhibiting intermittency between these two stable regimes. This suggests that localized, bounded oscillations may arise naturally in long filaments.

In the absence of permanent cross-links, we find no decay length for steady extensions even when active motors form temporary cross links. When permanent elastic links are present, the decay length depends on the extensional modulus of the filament and the shear modulus due to the permanent cross-links. Consistent with theoretical predictions, our simulations show that the ability of attached motors to sense local extension rates influences the motor activity and modifies the effective length scale over which strain decays. While the numerical model shows deviations from noiseless mean field predictions due to the presence of strong discrete noise caused by the variations in individual motor activity, several qualitative features are surprisingly still retained. Specifically, for fixed motor attachment and detachment rates, the decay length is set by the ratio of the passive elastic modulus of the constituent filament to the active shear modulus generated by attached motors - even in the presence of noise. This confirms a finite range for correlated activity that might be relevant for natural examples of ordered active matter such as eukaryotic flagella (4) by setting a limit over which mechanical coordination can persist. It also raises interesting questions about how to generalize our analysis to the deformations of bio-filaments in disordered mixtures of motors and cytoskeletal filaments $(46,47)$, particularly in the limit when the coordination number is small and when it approaches the isostatic limit. Our results also are relevant to understanding the mechanisms by which motor activity affects biofilament 
Effective elasticity and persistence of strain in active filament-motor assemblies

networks and solutions $(45,48,49)$, in evaluating persistence lengths in synthetic active systems (50-52), and in understanding how the decay of substrate strain induced due to contractility may coordinate the motions of cells $(54,55)$.

\section{AUTHOR CONTRIBUTIONS}

$\mathrm{AG}, \mathrm{RC}$ and LM designed the research and formulated the research questions. AG formulated the mean field theories for active and passive composites. RC formulated the stochastic MPC model and carried out all simulations. AG, RC and LM analyzed the data and wrote the paper.

\section{ACKNOWLEDGMENTS}

AG would like to acknowledge partial support from NSF via grant NSF-MCB-2026782.

\section{REFERENCES}

1. J. Howard, Mechanics of motor proteins and the cytoskeleton, (Sinauer Associates), ISBN 0878933344.

2. G. B. Witman, Introduction to cilia and flagella in ciliary and flagellar membranes (ed. R. A. Bloodgood), Plenum, New York, pp 1-30 (1990).

3. J. Howard (2008) Molecular mechanics of cells and tissues. Cell. Mol. Bioeng., 1(1): 24-32.

4. D. M. Woolley (2007) A novel motility pattern in quail spermatozoa with implications for the mechanism of flagellar beating. Biol. Cell, 99(12), 663-675.

5. R. Everaers, R. Bundschuh and K. Kremer (1995) Fluctuations and stiffness of double-stranded polymers: railway-track model. Europhys. Lett., 29 (3): 263-268.

6. L. Scharrel, R. Ma, R. Schneider, F. Jülicher and S. Diez (2014) Multimotor Transport in a System of Active and Inactive Kinesin-1 Motors. Biophys. J., 107:365-372.

7. C. Heussinger, M. Bathe and E. Frey (2007) Statistical mechanics of semiflexible bundles of wormlike chains. Phys. Rev. Lett., 99(4): 048101.

8. H. Mohrbach and I. M. Kulic (2007) Motor driven microtubule shape fluctuations: force from within the lattice. Phys. Rev. Lett., 99(21): 218102.

9. A.Vilfan and E. Frey (2005) Oscillations in molecular motor assemblies. J. Phys. Condens. Matter, 17(47):S3901-S3911.

10. S. W. Grill, K. Kruse and F. Jülicher (2005) Theory of mitotic spindle oscillations. Phys. Rev. Lett., 94(10): 108104.

11. E. Evans, K. Ritchie (1997) Dynamic strength of molecular adhesion bonds. Biophys. J., 72(4):1541-1555.

12. F. Gittes, B. Mickey, J. Nettleton and J. Howard (1993) Flexural rigidity of microtubules and actin filaments measured from thermal fluctuations in shape. J. Cell Biol., 120(4): 923-934.

13. C. J. Brokaw (1975) Molecular mechanism for oscillation in flagella and muscle. Proc. Natl. Acad. Sci. USA, 72(8): 3102-3106.

14. C. J. Brokaw (1989) Direct measurements of sliding between outer doublet microtubules in swimming sperm flagella. Science, 243(4898):1593-1596.

15. C. J. Brokaw (1971) Bend propagation by a sliding filament model for flagella. J. Exp. Biol., 55:289-304.

16. H. Kojima, M. Kikumoto, H. Sakakibara, K. and Oiwab (2002) Mechanical properties of a single-headed processive motor, inner-arm dynein subspecies-c of chlamydomonas studied at the single molecule level. J. Biolog. Phys., 28(3): 335-345.

17. H. Sui and K.H. Downing (2006) Molecular architecture of axonemal microtubule doublets revealed by cryo-electron tomography. Nature, 442(7101): 475-478.

18. K.E. Machin (1963) The control and synchronization of flagellar movement. Proc. Roy. Soc. B., 158(970), 88-104. 
Arvind Gopinath and Raghunath Chelakkot and L Mahadevan

19. V. Mukundan, P. Sartori, V. F. Geyer, F. Jülicher, and J. Howard (2014) Motor regulation results in distal forces that bend partially disintegrated chlamydomonas axonemes into circular arcs. Biophys. J., 106:1-9.

20. B. Qin, A. Gopinath, J. Yang, J. P. Gollub, and P. E. Arratia (2015) Flagellar kinematics and swimming of algal cells in viscoelastic fluids. Scientific Reports 5.

21. H. Yoke and C. Shingyogi (2017) Effects of external stress on the regulation of microtubule sliding induced by outer arm dynein of sea urchin sperm flagella. J. Exp. Biol., 220(6): 1122-1134.

22. M. J. Wargo, M. A. McPeek and E. F. Smith (2004) Analysis of microtubule sliding patterns in Chlamydomonas flagellar axonemes reveal dynein activity on specific doublet microtubules. J Cell Sci., 117: 2533-2544.

23. P. Satori, N. F. Geyer, A. Scholich, F. Julicher and J. Howard (2016) Dynamic curvature regulation accounts for the symmetric and asymmetric beats of Chlamydomonas flagella. eLife 5: e13258.

24. D. M. Woolley (2010) Flagellar oscillation: a commentary on proposed mechanisms. Biol. Rev. Camb. Philos. Soc.. 85: 453-470.

25. P. Satir, T. Heuser, W. S. Sale (2014) A structural basis for how motile cilia beat. BioScience, 64: 1073- 1083.

26. S. M. King (2018) Turning dyneins off bends cilia. Cytoskeleton, 75: 372-381.

27. Y. Man, F. Ling and E. Kanso (2019) Cilia oscillations. Phil. Trans. Roy. Soc. B, 375: 20190157.

28. J. Lin, K. Okada, M. Raytchev, M. C. Smith and D. Nicastro (2014) Structural mechanism of the dynein power stroke. Nat. Cell Biol., 16: 479.

29. J. Lin J and D. Nicastro (2018) Asymmetric distribution and spatial switching of dynein activity generates ciliary motility. Science, 360 (6387): eaar1968.

30. K. W. Foster, J. Vidyadharan and A. Sangani (2017) Evidence for a self-organized compliant mechanism for the spontaneous steady beating of cilia. Cell Motil. and Cytoskel., 74(7): 260-280.

31. R. Coy and H. Gadêlha (2017) The counterbend dynamics of cross-linked filament bundles and flagella. J. R. Soc. Interface, 14: 20170065.

32. M. Bottier, K. A. Thomas, S. K. Dutcher and P. V. Bayly (2019) How does cilium length affect beating? Biophys. J., 116: 1292-1304.

33. P. V. Bayly and K.S. Wilson (2014) Equations of interdoublet separation during flagella motion reveal mechanisms of wave propagation and instability. Biophysical J. 107, 1756.

34. P. V. Bayly and S. K. Dutcher (2016) Steady dynein forces induce flutter instability and propagating waves in mathematical models of flagella. J. Royal Soc. Interface 13, 20160523.

35. M. Bathe, C. Heussinger, M. M. Claessens, A. R. Bausch and E. Frey (2008) Cytoskeletal bundle mechanics. Biophys. J., 94(8): 2955.

36. C. Heussinger, F. Schüller and E. Frey (2010) Statics and dynamics of wormlike bundle model. Phys. Rev. E., 81(2): 021904.

37. A. Ward, F. Hilitski, W. Schwenger, D. Welch, A. W. C. Lau, V. Vitelli, L. Mahadevan and Z. Dogic (2015) Solid friction between soft filaments. Nat. Mater., 14, 583-588.

38. E. Reissner (1946) Analysis of shear lag in box beams by the principle of minimum potential energy. Quart. J. of Applied Math., 4:268-278.

39. K. Hatch, C. Danilowicz, V. Colijee and M. Prentiss (2008) Demonstration that the shear force required to separate short double-stranded DNA does not increase significantly with sequence length for sequences longer than 25 base pairs. Phys. Rev. E, 78(1):011920.

40. C. B. Lindemann, L. J. Macauley and K. A. Lesich, (2005) The counterbend phenomenon in dynein-disabled rat sperm flagella and what it reveals about the interdoublet elasticity. Biophys. J., 89(2):1165-1174. 
41. S. Camalet and F. Jülicher (2000) Generic aspects of axonemal beating. New J. Phys., 2: 24.1-24.23.

42. I. H. Riedel-Kruse, A. Hilfinger, J. Howard and F. Julicher (2007) How molecular motors shape the flagellar beat. HFSP Journal, 1(3):192-208.

43. G. Gompper, T. Ihle, D. M. Kroll and R. G. Winkler (2008) Multi-particle collision dynamics: a particle-based mesoscale simulation approach to the hydrodynamics of complex fluids. Adv. Polym. Sci., 221:1-87.

44. R. Chelakkot, R. G. Winkler and G. Gompper (2010) Migration of semiflexible polymers in microcapillary flow. Eur.Phys.Lett., 91: 14001.

45. A. E. Patteson, A. Gopinath and P. E. Arratia (2018). The propagation of active-passive interfaces in bacterial swarms Nature Communications, 9 (1), 5373.

46. T. Sanchez, D. Welch, D. Nicastro and Z. Dogic (2001) Cilia-like beating of active microtubule bundles. Science, 333(6041): 456-459.

47. T. Sanchez, D. T. N. Chen, S. J. DeCamp, M. Heymann and Z. Dogic (2012) Spontaneous motion in hierarchically assembled active matter. Nature, 491(7424): 431-434.

48. C. P. Broedersz and F. C. Mackintosh (2011) Molecular motors stiffen non-affine semiflexible polymer networks. Soft Matter, 7:3186.

49. C. A. Weber, R. Suzuki, V. Schaller, I. S. Aranson, A. R. Bausch and E. Frey (2015) Random bursts determine dynamics of active filaments. PNAS 112(34):10703-10707.

50. C. Li, B. Qin, A. Gopinath, P. E. Arratia, B. Thomases and R. D. Guy (2017) Flagellar swimming in viscoelastic fluids: role of fluid stress revealed by simulations based on experimental data. Proc. Roy. Soc. Interface., 14(135): 20170289.

51. S. Fatehiboroujeni, A. Gopinath and S. Goyal (2021) Three-dimensional nonlinear dynamics of prestressed active filaments: flapping, swirling, and flipping. Physical Review E 103 (1), 013005.

52. X. Liao, P.K. Purohit and A. Gopinath (2020) Extensions of the worm-like-chain model to tethered active filaments under tension. Journal of Chemical Physics 153, 194901

53. C. Bouchiat, M. D. Wang, A.-F. Allemand, T. Strick, S. M. Block and V. Croquette (1999) Estimating the Persistence Length of a Worm-Like Chain Molecule from Force-Extension Measurements. Biophysical J., 76:409-413.

54. S. Bose, K. Dasbiswas and A. Gopinath (2021) Matrix Stiffness Modulates Mechanical Interactions and Promotes Contact between Motile Cells. Biomedicines 9:428.

55. S. Goren, Y. Koren, X. Xu and A. Lesman (2020) Elastic anisotropy governs the range of cell-induced displacements. Biophysical Journal 118: 1152-1164.

\section{SUPPLEMENTARY MATERIAL}

Electronic supplementary files available as separate document 